\title{
On the global stability of an iterative scheme in a probabilistic Menger space
}

M De la Sen ${ }^{1 *}$ and A Ibeas ${ }^{2}$

\begin{abstract}
This paper studies the convergence properties of an iterative process which involves sequences of convergent self-mappings in probabilistic Menger spaces which are used to generate the sequences of interest. The convergent self-mappings under consideration satisfy conditions of either uniform or point-wise convergence, in a probabilistic sense, to a self-mapping on the same abstract space of the considered probabilistic metric space. Furthermore, the self-mappings of the considered sequence satisfy a probabilistic $\phi$-contractive condition which is based on the use of a control $\phi$-function. Some illustrative examples are also discussed.
\end{abstract}

\section{Introduction}

Fixed point theory [1-4] is receiving important research attention in the framework of probabilistic metric spaces. See, for instance, [2-6] and [7-11]. On the other hand, Menger probabilistic metric spaces are a special case of the wider class of probabilistic metric spaces which are endowed with a triangular norm, [2, 3, 5, 7, 9, 12, 13]. In probabilistic metric spaces, and under an intuition-based point of view, the deterministic notion of distance is considered to be probabilistic in the sense that, given any two points $x$ and $y$ of a metric space, a measure of the distance between them is a probabilistic metric $F_{x, y}(t)$, rather than the deterministic distance $d(x, y)$, which is interpreted as the probability of the distance between $x$ and $y$ being less than $t(t>0)$ [3].

Fixed point theorems in complete Menger probabilistic metric spaces for probabilistic concepts of $B$ and $C$-contractions can be found in [2] together with a new notion of contraction, referred to as $(\Psi, C)$-contraction. Such a contraction was proved to be useful for multivalued mappings while it generalizes the previous concept of $C$-contraction. On the other hand, 2-cyclic $\phi$-contractions on intersecting subsets of complete Menger spaces were discussed in [5] for contractions based on control $\phi$-functions. See also [6]. It was found that fixed points are unique. Also, $\phi$-contractions in complete probabilistic Menger spaces have also been studied in [9] through the use of altering distances while probabilistic Banach spaces versus fixed point theory have been discussed in [8]. The concept of probabilistic complete metric space was adapted to the formalism of Banach spaces endowed with norms being defined by triangular functions and under a suitable ordering in the considered space. In parallel, mixed monotone operators in such Banach spaces were discussed while the existence of coupled minimal and maximal fixed points for these operators was analyzed and discussed in detail. Further extensions to contractive mappings 
in complete fuzzy metric spaces by using generalized distribution functions have been studied in $[6,7]$ and references therein. The concept of altering distances was exploited in a very general context to derive fixed point results in [14], and extended later on in [12] to Menger probabilistic metric spaces. On the other hand, some interesting general fixed point theorems have been very recently obtained in [13] for two new classes of contractive mappings in Menger probabilistic metric spaces. The results have been established for $\alpha-\psi$-contractive mappings and for a generalized $\beta$-type one as well. It also has to be pointed out that the parallel background literature related to best proximity points and fixed points in cyclic mappings in metric and Banach spaces is exhaustive. See, for instance, [15-25] and the references therein. It can be pointed out that, in a general context, fixed point theory is of special relevance to investigate properties such as the stability of dynamic continuous-time and discrete-time systems and convergence of trajectories to local or global equilibrium points. The related analysis is an alternative to Lyapunov or H-infinity-based stability tools [26-29].

This paper investigates an iterative scheme in a probabilistic metric Menger space in the following situations: (a) The iterative scheme is of a polytopic-type in the sense that the considered sequence of self-mappings is built with iteration-dependent weighting scalar sequences based on a predefined basic self-mapping on a certain normed space and a set of associate composite self-mappings if a probabilistic $\phi$-contractive condition is satisfied by each of the self-mappings of the sequence. The given basic self-mapping used to build the sequence of self-mappings which generate the iterative scheme is not assumed to be necessarily contractive. The obtained results are extended to the case when the contractive constraint is satisfied only for a certain subsequence of the generated solution of the iterative scheme,

(b) The iterative scheme is run by a sequence of $\phi$-contractive self-mappings which are not built based on the basic self-mapping of the above situation. It is assumed that the sequence converge uniformly or point-wise to a self-mapping on the same abstract set. In the first case, it is assumed that the limit self-mapping is $\phi$-contractive, while in the second one it is assumed that the members of the sequence of self-mappings of the iterative scheme are $\phi$-contractive.

\subsection{Notation}

$$
\begin{array}{ll}
\mathbf{R}_{+}=\{z \in \mathbf{R}: z>0\}, & \mathbf{R}_{0+}=\{z \in \mathbf{R}: z \geq 0\}, \\
\mathbf{Z}_{+}=\{z \in \mathbf{Z}: z>0\}, & \mathbf{Z}_{0+}=\{z \in \mathbf{Z}: z \geq 0\}, \quad \bar{n}=\{1,2, \ldots, n\},
\end{array}
$$

$\left\{T_{n}\right\}_{\rightarrow}^{\rightarrow} T^{*}$ (that is, $\limsup _{n \rightarrow \infty}\left\{\left\|T_{n} x-T^{*} x\right\|: x \in \operatorname{Dom} T_{n}\right\}=0 ; \forall x \in \operatorname{Dom} T$ ) and $\left\{T_{n}\right\} \rightarrow T^{*}$ (that is, $\lim _{n \rightarrow \infty} T_{n} x=T^{*} x ; \forall x \in \operatorname{Dom} T_{n}$ ) for $T^{*}, T_{n}: X \rightarrow X ; \forall n \in \mathbf{Z}_{0+}$ denote, respectively, uniform and point-wise convergence in $X$ of $T_{n}: X \rightarrow X$ to $T^{*}: X \rightarrow X$ provided that all of them have the same domain.

Fix $(T)$ denotes the set of fixed points of $T: X \rightarrow X$ and $\bar{k}=\{1, \ldots, k\}$.

The convergence of a sequence to a limit $\left\{x_{n}\right\} \rightarrow x$ is understood in a probabilistic sense, that is, $F_{x_{n}, x}(t) \rightarrow 1$ as $n \rightarrow \infty, \forall t \in \mathbf{R}_{+}$, where $F_{x, y}$ denotes a probability density function taking values in $[0,1]$ for every $(x, y) \in X \times X$, where $X$ is some appropriate abstract set. 


\section{Preliminaries}

Denote by $\mathbf{L}$, the set of distribution functions $F: \mathbf{R} \rightarrow[0,1]$ which are non-decreasing and left continuous such that $F(0)=0$ and $\sup _{t \in \mathbf{R}} F(t)=1$. Let $X$ be a nonempty set and let $F: X \times X \rightarrow \mathbf{L}$ be a mapping from $X \times X$, where $X$ is an abstract set of elements, into the set of distribution functions $\mathbf{L}$ which are symmetric functions of elements $F_{x, y}$ for every $(x, y) \in X \times X$, referred to as the probabilistic metric (or probability density). Then the ordered pair $(X, F)$ is a probabilistic metric space (PM) [1-3] if:

(1) $\forall x, y \in X \quad\left(\left(F_{x, y}(t)=1 ; \forall t \in \mathbf{R}_{+}\right) \Leftrightarrow(x=y)\right)$,

(2) $F_{x, y}(t)=F_{y, x}(t), \quad \forall x, y \in X, \forall t \in \mathbf{R}$,

(3) $\forall x, y, z \in X, \forall t_{1}, t_{2} \in \mathbf{R}_{+} \quad\left(\left(F_{x, y}\left(t_{1}\right)=F_{y, z}\left(t_{2}\right)=1\right) \Rightarrow\left(F_{x, z}\left(t_{1}+t_{2}\right)=1\right)\right)$.

Note that an interpretation is that $F: X \times X \rightarrow \mathbf{L}$ is a set of distribution functions. A particular distribution function $F_{x, y}$ is a probabilistic metric (or distance), which takes values $F_{x, y}(t)=H(t)$ identified with a mapping $H: \mathbf{R} \rightarrow[0,1]$ in the set of all the distribution functions $\mathbf{L}$, is denoted a probabilistic metric which is a mapping from $X \times X$ to a probability density function $F: \mathbf{R} \rightarrow[0,1]$.

A Menger PM space is a triplet $(X, F, \Delta)$, where $(X, F)$ is a PM space which satisfies

$$
F_{x, y}\left(t_{1}+t_{2}\right) \geq \Delta\left(F_{x, z}\left(t_{1}\right), F_{z, y}\left(t_{2}\right)\right), \quad \forall x, y \in X, \forall t_{1}, t_{2} \in \mathbf{R}_{0+},
$$

under $\Delta:[0,1] \times[0,1] \rightarrow[0,1]$ is a $t$-norm (or triangular norm) belonging to the set $\mathbf{T}$ of $t$-norms which satisfy the properties:

(1) $\Delta(a, 1)=a$,

(2) $\Delta(a, b)=\Delta(b, a)$,

(3) $\Delta(c, d) \geq \Delta(a, b)$ if $c \geq a, d \geq b$,

(4) $\Delta(\Delta(a, b), c)=\Delta(a, \Delta(b, c))$.

Note that above properties imply that $\Delta(a, 0)=0$. The (probabilistic) diameter of a subset $A$ of $X$ is defined by $D_{A}^{p 0}(z)=\sup _{t<z} \inf _{x, y \in A} F_{x, y}(t)$ and $A$ is probabilistically bounded if $D_{A}^{p}=\sup _{z \in \mathbf{R}_{+}} D_{A}^{0}(z)=1$ [2]. Note that the diameter of a set refers to the real interval length where the argument of the probabilistic metric is nonzero while the probabilistic diameter is a measure of boundedness or unboundedness of such a set. The (probabilistic) distance in-between the subsets $A$ and $B$ of $X$ defines the argument interval length of zero probability distance in-between points of two subsets $A$ and $B$ of $X$ and it is defined as

$$
D=d(A, B)=\inf \left(z \in \mathbf{R}_{0+}: \sup _{x \in A, y \in B} F_{x, y}(t)=0 ; \forall t \in[0, z]\right) .
$$

Definition 2.1 [5, 6] A function $\phi: \mathbf{R} \rightarrow \mathbf{R}_{0+}$ is said to be a $\Phi$-function if it satisfies the conditions:

(1) $\phi(t)=0$ if and only if $t=0$,

(2) $\phi: \mathbf{R} \rightarrow \mathbf{R}_{0+}$ is strictly increasing,

(3) $\phi: \mathbf{R} \rightarrow \mathbf{R}_{0+}$ is left continuous in $\mathbf{R}_{+}$and continuous at $t=0$. 
Definition 2.2 [5, 6] Let $(X, F, \Delta)$ be a Menger space. A self-mapping $T: X \rightarrow X$ is $\phi$ contractive for some $\Phi$-function $\phi: \mathbf{R} \rightarrow \mathbf{R}_{0+}$ if for some $K(<1) \in \mathbf{R}_{+}$

$$
F_{T x, T y}(\phi(t)) \geq F_{x, y}\left(\phi\left(K^{-1} t\right)\right), \quad \forall t \in \mathbf{R}_{+} \text {for any for any } x, y \in X \text { and some } K \in(0,1) .
$$

Definition 2.3 [1,2] A sequence $\left\{x_{n}\right\} \subset X$ converges to $x \in X$ if, for any given real constants $\varepsilon, \lambda \in \mathbf{R}_{+}$, there is $n_{0}=n_{0}(\varepsilon, \lambda) \in \mathbf{Z}_{0+}$ such that $F_{x_{n}, x}(\varepsilon)>1-\lambda$ for $n>n_{0}$.

Definition 2.4 $[1,2]$ A sequence $\left\{x_{n}\right\}$ is a Cauchy sequence in $X$ if, for any given real constants $\varepsilon, \lambda \in \mathbf{R}_{+}$, there is $n_{0}=n_{0}(\varepsilon, \lambda) \in \mathbf{Z}_{0+}$ such that $F_{x_{n}, x_{m}}(\varepsilon) \geq 1-\lambda$ for $n, m>n_{0}$.

Note that if $\left\{x_{n}\right\} \subset S$ converges to $x \in S$ then $\lim _{n \rightarrow \infty} F_{x_{n}, x}(t)=1, \forall t \in \mathbf{R}_{+}$and if $\left\{x_{n}\right\}$ is a Cauchy sequence in $X$ then $\lim _{n, m \rightarrow \infty} F_{x_{n}, x_{m}}(t)=1, \forall t \in \mathbf{R}_{+}$. A Menger PM space $(X, F, \Delta)$ is complete if every Cauchy sequence is convergent in $X$.

\section{Iterative scheme and its properties}

Now, consider the following iterative scheme under a sequence of self-mappings $T_{n}: X \rightarrow$ $X, \forall n \in \mathbf{Z}_{0_{+}}$, on a vector space $X$ in a probabilistic Menger space $(X, F, \Delta)$ :

$$
x_{n+1}=T_{n} x_{n}, \quad \forall n \in \mathbf{Z}_{0+}
$$

for any given $x_{0} \in X$ with $T: X \rightarrow X$ and $T_{n}: X \rightarrow X ; \forall n \in \mathbf{Z}_{0+}$ being defined by $x_{n+1}=$ $T_{n} x=\left(\sum_{i=0}^{k} \alpha_{i}^{(n)} T^{i}\right)$ for any $x \in X$, and the nonnegative real parameterization sequences being subject to $\sum_{i=0}^{k} \alpha_{i}^{(n)}>0 ; \forall i \in \bar{k}=\{0,1, \ldots, k\}, \forall n \in \mathbf{Z}_{0+}$. A main result of the paper which revisits with a probabilistic approach one of the deterministic iterative schemes discussed in [30] follows in the sequel.

Theorem 3.1 Consider the special iterative scheme (3.1) of the form $T_{n} x=\left(\sum_{i=0}^{k} \alpha_{i}^{(n)} T^{i}\right) x$ for any $x \in X$, on a complete Menger space $\left(X, F, \Delta_{M}\right)$, endowed with the minimum triangular norm $\Delta_{M}$, where $(X,\|\|)$ is an associated normed vector space, under the following assumptions:

(1) $\sum_{i=0}^{k} \alpha_{i}^{(n)}>0$ and $0 \leq \alpha_{i}^{(n+1)}=\alpha_{i}^{(n)}+\tilde{\alpha}_{i}^{(n)} ; \forall i \in \bar{k}=\{0,1, \ldots, k\}, \forall n \in \mathbf{Z}_{0+}$ with the nonnegative real sequences $\left\{\alpha_{i}^{(n)}\right\} ; \forall i \in \bar{k}$ being subject to the constraints

$$
\begin{aligned}
& \left|\tilde{\alpha}_{i}^{(n)}\right| \leq \tilde{\alpha}_{n} \leq m_{n} \frac{\left\|x_{n}-x_{n+1}\right\|}{\left\|x_{n+1}\right\|}, \\
& m_{n} \leq \frac{1-\rho}{k+1} \frac{\left\|x_{n+1}\right\|}{\left\|x_{n+1}-x_{n}\right\|} ; \quad \forall i \in \bar{k}, \forall n \in \mathbf{Z}_{0+} ;
\end{aligned}
$$

(2)

$$
F_{x_{n+2}, x_{n+1}}(\phi(t)) \geq F_{x_{n+1}, x_{n}}\left(\phi\left(\rho^{-1} t\right)\right), \quad \forall t \in \mathbf{R}_{+}, \forall n \in \mathbf{Z}_{0+},
$$

for some $\rho \in(0,1)$ and some $\Phi$-function $\phi: \mathbf{R} \rightarrow \mathbf{R}_{0+}$.

Then the following properties hold:

(i) There exists the limit $\lim _{n \rightarrow \infty} F_{x_{n}, x_{n+1}}(t)=1, \forall t \in \mathbf{R}_{+}$, for any given initial point $x_{0} \in X$ of the iterative scheme (3.1) and the sequence $\left\{x_{n}\right\}$ is bounded. 
(ii) If $T_{n} 0=0, \forall n \in \mathbf{Z}_{0+}$ then $x=0$ is the unique equilibrium point of the iterative scheme $x_{n+1}=T_{n} x=\left(\sum_{i=0}^{k} \alpha_{i}^{(n)} T^{i}\right) x_{n}$ for any $x_{0} \in X$ and also a fixed point of $T_{n}: X \rightarrow X$, $\forall n \in \mathbf{Z}_{0+}$, to which any sequence solution $\left\{x_{n}\right\} \subset X$, which is furthermore a Cauchy sequence, converges for any given initial point $x_{0} \in X$.

Proof Define the $(k+1)$ error sequences $\left\{\tilde{\alpha}_{i}^{(n)}\right\}$ by $\tilde{\alpha}_{i}^{(n)}=\alpha_{i}^{(n+1)}-\alpha_{i}^{(n)} ; \forall i \in \bar{k}=\{0,1, \ldots, k\}$, $\forall n \in \mathbf{Z}_{0+}$. If $(X,\|\|)$ is a normed vector space then there is always a metric-induced norm $d(x, y)=\|x-y\|, \forall x, y \in X$. Thus, one finds from the property of a complete Menger space $\left(X, F, \Delta_{M}\right),(2.2),(3.1), \sum_{i=0}^{k} \alpha_{i}^{(n)}>0$, and $0 \leq \alpha_{i}^{(n+1)}=\alpha_{i}^{(n)}+\tilde{\alpha}_{i}^{(n)} ; \forall i \in \bar{k}=\{0,1, \ldots, k\}, \forall n \in$ $\mathrm{Z}_{0+}$, that

$$
\begin{aligned}
& F_{x_{n+2}, x_{n+1}}(\phi(t)) \\
&= F_{\sum_{i=0}^{k}\left(\alpha_{i}^{(n)} T^{i} x_{n+1}+\tilde{\alpha}_{i}^{(n)} T^{i} x_{n+1}\right), \sum_{i=0}^{k} \alpha_{i}^{(n)} T^{i} x_{n}}(\phi(t)) \\
& \geq \Delta_{M}\left(F_{\sum_{i=0}^{k} \alpha_{i}^{(n)} T^{i} x_{n+1}, \sum_{i=0}^{k} \alpha_{i}^{(n)} T^{i} x_{n}}(\phi(t) / 2),\right. \\
&\left.F_{\sum_{i=0}^{k} \alpha_{i}^{(n)} T^{i} x_{n+1}+\tilde{\alpha}_{i}^{(n)} T^{i} x_{n+1}, \sum_{i=0}^{k} \alpha_{i}^{(n)} T^{i} x_{n+1}}(\phi(t) / 2)\right) \\
&= \Delta_{M}\left(F_{\sum_{i=0}^{k} \alpha_{i}^{(n)} T^{i} x_{n+1}, \sum_{i=0}^{k} \alpha_{i}^{(n)} T^{i} x_{n}}(\phi(t) / 2),\right. \\
&\left.F_{\sum_{i=0}^{k} \tilde{\alpha}_{i}^{(n)} T^{i} x_{n+1}}(\phi(t) / 2)\right), \quad \forall t \in \mathbf{R}_{+}, \forall_{n} \in Z_{0+} .
\end{aligned}
$$

The last equality in (3.4) arises for any given $a, b \in X$, since $(X,\|\|)$ is a normed vector space, one has trivially $\|a+b-a\|=\|b\|$ and, since $0 \in X$, it follows that $F_{a+b, a}(t)=F_{b, 0}(t)$, $\forall t \in \mathbf{R}_{+}$, in the Menger PM space $\left(X, F, \Delta_{M}\right)$. Otherwise, the probability of $\|b\|$ to be less than some $t \in \mathbf{R}_{+}$would be distinct to the probability of $\|a+b-a\|$ to be less than such a positive real value $t$, which is impossible. In the same way and, since $\|a+b+c-d-e-f\|=$ $\|a-(d+e+f-b-c)\|$ for any given $a, b, c, d, e, f \in X$, it follows that

$$
\begin{aligned}
F_{a+b+c, d+e+f}(t) & =F_{a, d+e+f-b-c}(t) \\
& \geq \Delta_{M}\left(F_{a, d}(t / 2), F_{d, d+e+f-b-c}(t / 2)\right) \\
& \geq \Delta_{M}\left(F_{a, d}(t / 2), F_{b+c, e+f}(t / 2)\right) \\
& =\Delta_{M}\left(F_{a, d}(t / 2), \Delta_{M} F_{b, e+f-c}(t / 2)\right) \\
& \geq \Delta_{M}\left(F_{a, d}(t / 2), \Delta\left(F_{b, e}(t / 4) F_{e, e+f-c}(t / 4)\right)\right) \\
& =\Delta_{M}\left(F_{a, d}(t / 2), \Delta_{M}\left(F_{b, e}(t / 4) F_{c, f}(t / 4)\right)\right), \quad \forall t \in \mathbf{R}_{+},
\end{aligned}
$$

which becomes for $c=d=e=0$ and $f=\sum_{i=0}^{k} f_{i}$ and the symmetry of the probability density function (second property of (2.1)), the associative property of the triangular norms (fourth property of (2.3)) and the use of the minimum triangular norm:

$$
\begin{aligned}
& F_{a+f, b}(t) \\
& \quad \geq \Delta_{M}\left(F_{a, 0}(t / 2), \Delta_{M}\left(F_{b, 0}(t / 4), F_{f, 0}(t / 4)\right)\right) \\
& \quad=\Delta_{M}\left(F_{\sum_{i=0}^{k} f_{i}, 0}(t / 4), \Delta_{M}\left(F_{a, 0}(t / 2), F_{b, 0}(t / 4)\right)\right) \\
& \quad \geq \Delta_{M}\left(\Delta_{M}\left(F_{f_{0}, 0}(t / 8), F_{\sum_{i=1}^{k} f_{i}, 0}(t / 8)\right), \Delta_{M}\left(F_{a, 0}(t / 2), F_{b, 0}(t / 4)\right)\right)
\end{aligned}
$$




$$
\begin{aligned}
& \geq \Delta_{M}\left(\Delta_{M}\left(F_{a, 0}\left(t /\left(2^{k+3}\right)\right), F_{b, 0}\left(t /\left(2^{k+3}\right)\right)\right), \min _{0 \leq i \leq k} F_{f_{i}, 0}\left(t /\left(2^{k+3}\right)\right)\right) \\
& \geq \min \left(\min \left[F_{a, 0}\left(t /\left(2^{k+3}\right)\right), F_{b, 0}\left(t /\left(2^{k+3}\right)\right)\right], \min _{0 \leq i \leq k}\left[F_{f_{i}, 0}\left(t /\left(2^{k+3}\right)\right)\right]\right) \\
& \geq \min \left(F_{a, 0}\left(t /\left(2^{k+3}\right)\right), F_{b, 0}\left(t /\left(2^{k+3}\right)\right), \min _{0 \leq i \leq k}\left[F_{f_{i}, 0}\left(t /\left(2^{k+3}\right)\right)\right]\right), \quad \forall t \in \mathbf{R}_{+},
\end{aligned}
$$

due to the fact that $F_{a, 0}(t / 2) \geq F_{a, 0}(t / 8), F_{b, 0}(t / 4) \geq F_{b, 0}(t / 8)$ since the distribution function is non-decreasing. Now, define auxiliary sequences.

$$
\begin{aligned}
& a=a(n)=\sum_{i=0}^{k} \alpha_{i}^{(n)} T^{i} x_{n+1}, \\
& b=b(n)=\sum_{i=0}^{k} \alpha_{i}^{(n)} T^{i} x_{n}, \\
& f_{i}=f_{i}(n)=\tilde{\alpha}_{i}^{(n)} T^{i} x_{n+1}, \quad f=f(n)=\sum_{i=0}^{k} \tilde{\alpha}_{i}^{(n)} T^{i} x_{n+1}=\sum_{i=0}^{k} f_{i},
\end{aligned}
$$

$\forall n \in \mathbf{Z}_{0+}$, so that one gets from (3.6)-(3.7) into (3.4) and since $0 \in X$ :

$$
\begin{aligned}
& F_{x_{n+2}, x_{n+1}}(\phi(t)) \\
& \geq \Delta_{M}\left(\Delta_{M}\left(F_{\sum_{i=0}^{k} \alpha_{i}^{(n)} T^{i} x_{n+1}, 0}\left(\phi\left(t /\left(2^{k+2}\right)\right)\right), F_{\sum_{i=0}^{k} \alpha_{i}^{(n)} T^{i} x_{n}, 0}\left(\phi\left(t /\left(2^{k+2}\right)\right)\right)\right),\right. \\
&\left.\quad \min _{0 \leq i \leq k} F_{\tilde{\alpha}_{i}^{(n)} T^{i} x_{n+1}, 0}\left(\phi\left(t /\left(2^{k+2}\right)\right)\right)\right) \\
& \geq \min \left(F_{x_{n+1}, 0}\left(\phi\left(t /\left(2^{k+3} \rho\right)\right)\right), F_{x_{n}, 0}\left(\phi\left(t /\left(2^{k+3} \rho\right)\right)\right),\right. \\
&\left.F_{x_{n+1}, 0}\left(\phi\left((k+1) t /\left(2^{k+3}(1-\rho)\right)\right)\right)\right), \quad \forall t \in \mathbf{R}_{+} .
\end{aligned}
$$

From (3.2)-(3.3) and $\left|\tilde{\alpha}_{n}\right| \leq 1-\rho$, one gets

$$
\min _{0 \leq i \leq k} F_{\tilde{\alpha}_{i}^{(n)} T^{i} x_{n+1}, 0}\left(\phi\left(t /\left(2^{k+3}\right)\right)\right) \geq F_{x_{n+1}, 0}\left(\phi\left((k+1) t /\left(2^{k+3}(1-\rho)\right)\right)\right), \quad \forall n \in \mathbf{Z}_{0+} .
$$

The use of the constraints (3.2)-(3.3), and the fact that $T_{n} 0=0 \in X, \forall n \in \mathbf{Z}_{0+}$, from (3.1) and $0 \in X$, yield

$$
\begin{aligned}
F_{x_{n+1}, 0}(\phi(t)) & =F_{\sum_{i=0}^{k} \alpha_{i}^{(n)} T^{i} x_{n}, 0}(\phi(t)) \\
\geq & F_{x_{n}, 0}\left(\phi\left(\rho^{-1} t\right)\right), \forall t \in \mathbf{R}_{+}, \quad \forall n \in \mathbf{Z}_{0+}, \\
F_{x_{n+2}, \sum_{i=0}^{k} \alpha_{i}^{(n)} T^{i} x_{n+1}}(\phi(t)) & =F_{\sum_{i=0}^{k} \tilde{\alpha}_{i}^{(n)} T^{i} x_{n+1}, 0}(\phi(t)) \\
& \geq F_{x_{n+1}, 0}\left(\phi\left(t /\left(2^{k+3}(1-\rho)\right)\right)\right) \\
& \geq F_{x_{n+1}, 0}\left(\phi\left(\rho^{-1} t\right)\right), \quad \forall t \in \mathbf{R}_{+}, \forall n \in \mathbf{Z}_{0+},
\end{aligned}
$$

for any sequence $\left\{x_{n}\right\} \subset X$ generated from (3.1) for any initial condition $x_{0} \in X$ since $\phi$ is strictly increasing and the probability density function is non-decreasing. The use of 
(3.10)-(3.11) in (3.8) yields by using recursive calculations:

$$
\begin{aligned}
F_{x_{n+1}, x_{n}}(\phi(t)) \\
\geq \min \left(F_{x_{n}, 0}\left(\phi\left(t /\left(2^{k+3} \rho\right)\right)\right), F_{x_{n-1}, 0}\left(\phi\left(t /\left(2^{k+3} \rho\right)\right)\right), F_{x_{n}, 0}\left(\phi\left((k+1) t /\left(2^{k+3}(1-\rho)\right)\right)\right)\right) \\
\quad \ldots \\
\geq \min \left(F_{x_{0}, 0}\left(\phi\left(t /\left(2^{k+3} \rho^{n+1}\right)\right)\right), F_{x_{0}, 0}\left(\phi\left(t /\left(2^{k+3} \rho^{n}\right)\right)\right),\right. \\
\left.\quad F_{x_{0}, 0}\left(\phi\left((k+1) t /\left(2^{k+3}(1-\rho)^{n+1}\right)\right)\right)\right), \quad \forall t \in \mathbf{R}_{+}, \forall n \in \mathbf{Z}_{+} .
\end{aligned}
$$

Since $\rho \in(0,1),(1-\rho) \in(0,1)$ and $\phi: \mathbf{R} \rightarrow \mathbf{R}_{0+}$ is a $\Phi$-function we have $\lim _{n \rightarrow \infty} \phi(t)$ $\left.\left(2^{k+3} \rho^{n}\right)\right)=+\infty$ so that one finds from (3.12) that the limit below exists:

$$
\begin{aligned}
& \lim _{n \rightarrow \infty} F_{x_{0}, 0}\left(\phi\left(t /\left(2^{k+3} \rho^{n+1}\right)\right)\right) \\
& \quad=\lim _{n \rightarrow \infty} F_{x_{0}, 0}\left(\phi\left(t /\left(2^{k+3} \rho^{n}\right)\right)\right) \\
& \quad=\lim _{n \rightarrow \infty} F_{x_{0}, 0}\left(\phi\left((k+1) t /\left(2^{k+3}(1-\rho)^{n+1}\right)\right)\right)=1, \quad \forall t \in \mathbf{R}_{+}
\end{aligned}
$$

since $F_{x, y}: \mathbf{R} \rightarrow[0,1]$ is non-decreasing and left continuous with $F(0)=0$ and $\sup _{t \in \mathbf{R}} F(t)=1$. Then $\lim _{n \rightarrow \infty} F_{x_{n+1}, x_{n}}(\phi(t))=1, \forall t \in \mathbf{R}_{+}$from (3.12)-(3.13). Also, one sees for any given $\varepsilon \in \mathbf{R}_{+}, \lambda \in(0,1) \cap \mathbf{R}_{+}$that there exists $n_{0}=n_{0}(\varepsilon, \lambda) \in \mathbf{Z}_{0+}$ such that, for all $n\left(\in \mathbf{Z}_{+}\right)>n_{0}$,

$$
\begin{aligned}
F_{x_{n+1}, x_{n}}(\varepsilon)= & F_{x_{n+1}, x_{n}}\left(\phi\left(t_{1}^{-}\right)\right) \\
\geq & \min \left(F_{x_{0}, 0}\left(\phi\left(t_{1} /\left(2^{k+3} \rho^{n+1}\right)\right)\right), F_{x_{0}, 0}\left(\phi\left(t_{1} /\left(2^{k+3} \rho^{n}\right)\right)\right),\right. \\
& \left.F_{x_{0}, 0}\left(\left((k+1) t /\left(2^{k+3}(1-\rho)^{n+1}\right)\right)\right)\right) \\
\geq & 1-\lambda
\end{aligned}
$$

from (3.12) since $\lim _{n \rightarrow \infty} F_{x_{n+1}, x_{n}}(\phi(t))=1, \phi: \mathbf{R} \rightarrow \mathbf{R}_{0+}$ is left continuous and strictly increasing, continuous at $t=0$ and $\phi(t)=0$ if and only if $t=0$, and then there exists $t_{1}=t_{1}(\varepsilon) \in \mathbf{R}_{+}$such that $\phi\left(t_{1}^{-}\right)=\lim _{t \rightarrow t_{1}^{-}} \phi(t)=\varepsilon$. Thus, $\left\{x_{n}\right\} \subset X$ converges to some $a \in X$ (Definition 2.3) and it is bounded as a result. Also, one has from (2.2) that, once having fixed any $\varepsilon \in \mathbf{R}_{+}, \lambda \in(0,1) \subset \mathbf{R}$ and for all integers $n, m>n_{0}=n_{0}(\varepsilon, \lambda)$, there exists $\lambda_{1}(\leq \lambda) \in(0,1) \subset \mathbf{R}$ such that, since the distribution function is non-decreasing,

$$
\begin{aligned}
F_{x_{n}, x_{m}}(\varepsilon) & \geq \Delta_{M}\left(F_{x_{n}, a}(\varepsilon / 2), F_{a, x_{m}}(\varepsilon / 2)\right) \\
& \geq \Delta_{M}\left(1-\lambda_{1}, 1-\lambda_{1}\right)=1-\lambda_{1} \geq 1-\lambda
\end{aligned}
$$

and $\left\{x_{n}\right\} \subset X$ is a Cauchy sequence (Definition 2.4) and then it is bounded. It is clear from (3.8) that $F_{x_{n}, 0}\left(\phi\left(t /\left(2^{k+3} \rho\right)\right)\right) \rightarrow 1, \forall t \in \mathbf{R}_{+}$as $n \rightarrow \infty$ so that $\left\{x_{n}\right\} \rightarrow 0$ from the first property in (2.1) of the PM space $(X, F, \Delta)$. Since $T_{n} 0=0, \forall n \in \mathbf{Z}_{0+}$, from (3.1), we find that $x=0$ is an equilibrium point of (3.1) and a fixed point of all the members of the sequence of self-mappings $\left\{T_{n}\right\}$. It is now proved that such an equilibrium is unique. Assume that the uniqueness fails and that there is $a \neq 0$ which is also an equilibrium point of (3.1). Since 
$\phi$ is strictly increasing and $\rho<1$, choose $\delta \in \mathbf{R}_{+}$, such that $\phi\left(\rho^{-1} t\right)-\delta>\phi(t)$, and define $\lambda_{1} \in(0,1)$ such that $\lambda_{1}=\lambda_{1}(t)=1-F_{a, 0}\left(\rho^{-1} t\right)$. Since $a \neq 0$ according to the first property of the PM spaces in (2.1), implies that $F_{a, 0}\left(\rho^{-1} t\right)<1$ from the first property of the probabilistic metric space $(X, F, \Delta)(2.1)$. Now, fix any $\varepsilon \in \mathbf{R}_{+}$and $\lambda\left(<\lambda_{1}\right) \in(0,1) \cap \mathbf{R}$ such that if some solution sequence of (3.1) $\left\{x_{n}\right\}(\subset X) \rightarrow a$ then there exists $n_{0}=n_{0}(\varepsilon, \lambda) \in \mathbf{Z}_{0+}$ such that for all $n, m\left(\in \mathbf{Z}_{+}\right)>n_{0}$, one has

$$
\begin{aligned}
1-\lambda_{1} & =F_{a, 0}\left(\phi\left(\rho^{-1} t\right)\right) \geq \Delta_{M}\left(F_{a, x_{n}}(\delta), F_{x_{n}, x_{m}}\left(\phi\left(\rho^{-1} t\right)-\delta\right)\right) \\
& >\Delta_{M}(1-\lambda, 1-\lambda)=1-\lambda,
\end{aligned}
$$

which contradicts the choice $\lambda<\lambda_{1}$ so that $x=0$ is the unique equilibrium point of (3.1) and trivially a fixed point of $T_{n}: X \rightarrow X$. This fixed point is unique. Assume that this is not the case. Then $T_{n} 0=0, \forall n \in \mathbf{Z}_{0+}$, and assume that for some $m \in \mathbf{Z}_{0+}, T_{m}^{n} x=T_{m} x=x \neq 0$. Thus, $F_{x, 0}(\varepsilon)=F_{T_{m}^{n} x, T_{m}^{n} 0}(\varepsilon) \geq F_{x, 0}\left(\phi\left(\rho^{-n} t\right)\right)$ for any given $\varepsilon \in \mathbf{R}_{+}$and a unique $t=t(\varepsilon) \in \mathbf{R}_{+}$ satisfying $\phi(t)=\varepsilon$, which exists since $\phi: \mathbf{R}_{0+} \rightarrow \mathbf{R}_{0+}$ is a $\Phi$-function. As a result,

$$
\liminf _{n \rightarrow \infty}\left(F_{x, 0}(\varepsilon)-F_{x, 0}\left(\phi\left(\rho^{-n} t\right)\right)\right)=F_{x, 0}(\varepsilon)-\lim _{t \rightarrow \infty} F_{x, 0}(t)=F_{x, 0}(\varepsilon)-1 \geq 0 .
$$

Then $F_{x, 0}(\varepsilon)=1$ so that $x=0$. Properties [(i)-(ii)] have been proved.

Three direct results follow from Theorem 3.1.

Corollary 3.1 Consider the special iterative scheme (3.1) of the form $T_{n} x=\left(\sum_{i=0}^{k} \alpha_{i}^{(n)} T^{i}\right) x$ for any $x \in X$, on a complete Menger space $\left(X, F, \Delta_{M}\right)$, endowed with the minimum triangular norm $\Delta_{M}$, such that $(X, d)$ is a metric space with $0 \in X$ and $d: X \times X \rightarrow \mathbf{R}_{0+}$ is a homogeneous and translation-invariant distance under the remaining assumptions of Theorem 3.1. Then Theorem 3.1 still holds.

Proof Since $d: X \times X \rightarrow \mathbf{R}_{0+}$ is a homogeneous and translation-invariant distance, there is a metric-induced norm \|\| such that $(X,\|\|) \equiv(X, d)$ is a normed space and the proof of Theorem 3.1 remains valid for a Menger PM space $(X, F, \Delta)$, where $(X, F)$ is a PM space.

Corollary 3.2 Consider the special iterative scheme (3.1) of the form $T_{n} x=\left(\sum_{i=0}^{k} \alpha_{i}^{(n)} T^{i}\right) x$ for any $x \in X$, on a complete Menger space $\left(X, F, \Delta_{M}\right)$, with $0 \in X$, endowed with the minimum triangular norm $\Delta_{M}$, such that the probability density function has the additional property $F_{x, y}(t)=F_{x+z, y+z}(t), \forall x, y, z \in X, \forall t \in \mathbf{R}_{0+}$. Then Theorem 3.1 still holds under the remaining given assumptions.

Proof The property $F_{x, y}(t)=F_{x+z, y+z}(t)=F_{0, y-x}(t), \forall x, y, z \in X, \forall t \in \mathbf{R}_{0+}$, since $0 \in X$ and $-x \in X$ since $x \in X$, allows one to follow the steps in the proof of Theorem 3.1 without the use of a distance or a norm for $X$.

Corollary 3.3 Assume that the self-mapping $T_{n}$ from $X$ to $X$ defined by $T_{n} x=T_{c} x=$ $\sum_{i=1}^{n} \alpha_{i}^{(n)} T^{i} x, \forall x \in X$, is independent of $n \in \mathbf{Z}_{0_{+}}$, which holds in particular if $\alpha_{i}^{(n)}=\alpha_{i}, \forall i \in \bar{k}$, $\forall n \in \mathbf{Z}_{0+}$, are real constants, and $T_{c} 0=0$. Assume also that the remaining assumptions of Theorem 3.1 hold. Then $x=0$ is the unique fixed point of $T_{c}$. 
Remark 3.1 The above results remain valid for a Menger space $\left(F, X, \Delta_{L}\right)$ defined under the Lukasiewicz triangular norm $\Delta_{L}(a, b)=\max (0, a+b-1)$ since $\Delta_{L}\left(a_{n}, b_{n}\right)=$ $\max \left(0, a_{n}+b_{n}-1\right) \rightarrow 1$ as $n \rightarrow \infty$, provided that $\left\{a_{n}\right\},\left\{b_{n}\right\} \rightarrow 1$ as $n \rightarrow \infty$, still yielding $\lim _{n \rightarrow \infty} F_{x_{n+1}, x_{n}}(\phi(t))=1, \forall t \in \mathbf{R}_{+}$, then (3.14)-(3.15) still hold for the Lukasiewicz triangular norm from (3.12)-(3.13), by replacing $1-\lambda \rightarrow 1-2 \lambda$ for any real constant $\lambda \in(0,1)$ being chosen for $\Delta_{M}$. The results remain valid also for a Menger space $\left(F, X, \Delta_{n M}\right)$ where $\Delta_{n M}$ is the nilpotent minimum triangular norm defined by $\Delta_{n M}(a, b)=\min (a, b)$ if $a+b>1$ and $\Delta_{n M}(a, b)=0$ if $a+b \leq 1$ since $\Delta_{n M}\left(a_{n}, b_{n}\right) \rightarrow 1, \Delta_{M}\left(a_{n}, b_{n}\right) \rightarrow 1$ as $n \rightarrow \infty$ in the case that $\left\{a_{n}\right\},\left\{b_{n}\right\} \rightarrow 1$ as $n \rightarrow \infty$. It is not difficult to see that the results are also valid for any triangular norm satisfying $\Delta(a, a) \geq a$.

Theorem 3.1 is reformulated under weaker conditions if the contractive condition holds for consecutive members of the subsequences generated by the iterative scheme.

Theorem 3.2 Let all the assumptions in Theorem 3.1 be kept identical except that the $\phi$ contractive condition is weakened to the form

$$
F_{\bar{x}_{n+2}, \bar{x}_{n+1}}(\phi(t)) \geq F_{\bar{x}_{n+1}, \bar{x}_{n}}\left(\phi\left(\rho^{-1} t\right)\right), \quad \forall t \in \mathbf{R}_{+}, \forall n \in \mathbf{Z}_{0+}
$$

for some $\Phi$-function $\phi: \mathbf{R} \rightarrow \mathbf{R}_{0+}$ and some real constant $\rho \in(0,1)$, where $\left\{\bar{x}_{n}\right\} \subset\left\{x_{n}\right\} \subset X$ is defined by $\bar{x}_{0}=x_{0}$ and $\bar{x}_{n}=x_{\sum_{i=0}^{n} m_{i}}, \forall n \in \mathbf{Z}_{0_{+}}$, and the sequence of nonnegative integers $\left\{m_{i}\right\}$ satisfies $m_{i+1}-m_{i} \leq M<+\infty, \forall i \in \mathbf{Z}_{0+}$.

Then the following properties hold:

(i) There exists the limit $\lim _{n \rightarrow \infty} F_{\bar{x}_{n}, \bar{x}_{n+1}}(t)=1, \forall t \in \mathbf{R}_{+}$, for any given initial point $\bar{x}_{0}=x_{0} \in X$ of the iterative scheme (3.1) and the sequence $\left\{\bar{x}_{n}\right\}$ is bounded.

(ii) Define the composite self-mapping $\hat{T}(n+m+1, n)=T_{n+m} \cdots T_{n+1} . T_{n}$ from $X$ to $X$, $\forall n, m \in \mathbf{Z}_{0+}$. If $\bar{T}_{n} 0=0, \forall n \in \mathbf{Z}_{0_{+}}$, then $\bar{x}=0$ is the unique equilibrium point of the iterative scheme $\bar{x}_{n+1}=\hat{T}(n+m+1, n) \bar{x}_{n}$ for any initial given point $\bar{x}_{0}=x_{0} \in X$ and it is also a fixed point of $\hat{T}(n+m+1, n), \forall n, m \in \mathbf{Z}_{0+}$, to which any sequence solution $\left\{\bar{x}_{n}\right\} \subset X$, which is furthermore a Cauchy sequence, converges for any given initial point $\bar{x}_{0}=x_{0} \in X$.

(iii) If, in addition to the $\phi$-contractive condition (3.18a), there is family of a uniformly bounded positive real constants $\rho_{n}\left(i_{n}, j_{n}\right)=\rho_{n}\left(m_{0}, m_{1}, \ldots, m_{n}, i_{n}, j_{n}\right)$, which are not necessarily less than unity, with $i_{n}, j_{n} \in \mathbf{Z}_{+}$being any integers satisfying $1<i_{n}<j_{n} \leq m_{n+1}, \forall n \in \mathbf{Z}_{0+}$, such that

$$
\begin{aligned}
& F_{\sum_{k=0}^{n+1} m_{k+j}+j_{n+1}, x_{\sum_{k=0}^{n+1} m_{k}+i_{n+1}}}(\phi(t)) \\
& \quad \geq F_{\bar{x}_{n+1}, \bar{x}_{n}}\left(\phi\left(\rho_{n}^{-1}\left(i_{n}, j_{n}\right) t\right)\right), \quad \forall t \in \mathbf{R}_{+}, \forall n \in \mathbf{Z}_{0+},
\end{aligned}
$$

then $x=0$ is the unique equilibrium point of the iterative scheme $x_{n+1}=T_{n} x=\left(\sum_{i=0}^{k} \alpha_{i}^{(n)} T^{i}\right) x_{n}$ for any $x_{0} \in X$ and also a fixed point of $\hat{T}(n+m+1, n)$, $\forall n, m \in \mathbf{Z}_{0_{+}}$, to which any sequence solution $\left\{x_{n}\right\} \subset X$, which is furthermore a Cauchy sequence, converges to the same limit $\bar{x}$ of its subsequence $\left\{\bar{x}_{n}\right\}$ for any given initial point $x_{0} \in X$. 
Proof Since $\left\{\bar{x}_{n}\right\} \subset\left\{x_{n}\right\} \subset X$ and the $\phi$-contractive condition (3.18a) holds for such a subsequence, the proof of Theorem 3.1 is valid for any subsequence $\left\{\bar{x}_{n}\right\}$ of $\left\{x_{n}\right\} \subset X$ generated by the iterative scheme. Hence, $\lim _{n \rightarrow \infty} F_{\bar{x}_{n}, \bar{x}_{n+1}}(t)=1,\left\{\bar{x}_{n}\right\}$ bounded, Cauchy, and convergent, $\bar{T}_{n} 0=0, \forall n \in \mathbf{Z}_{0+} \Rightarrow \bar{x}=0$ being the unique equilibrium point of the iterative scheme and the unique fixed point of the composite self-mapping $\hat{T}(n+m+1, n)$ on $X$.

To prove (iii), it remains to be proved that the properties of Part (ii) are inherited by $T_{n}: X \rightarrow X$ if both (3.18a)-(3.18b) hold. From (3.18b) into (3.18a), since $x_{0}=\bar{x}_{0}$,

$$
\begin{aligned}
& F_{x_{\sum_{k=0}^{n+1} m_{k}+j_{n+1}, x^{\prime}} \sum_{k=0}^{n+1} m_{k}+i_{n+1}}(\phi(t)) \\
& \quad \geq F_{\bar{x}_{n+1}, \bar{x}_{n}}\left(\phi\left(\rho_{n}^{-1}\left(i_{n}, j_{n}\right) t\right)\right) \\
& \quad \geq F_{x_{0}, \bar{x}_{1}}\left(\phi\left(\rho^{-n+1} \rho_{n}^{-1}\left(i_{n}, j_{n}\right) t\right)\right), \quad \forall t \in \mathbf{R}_{+}, \forall n \in \mathbf{Z}_{0+},
\end{aligned}
$$

and, since $\infty>\rho_{n}^{-1}\left(i_{n}, j_{n}\right)>0, \forall n \in \mathbf{Z}_{0+}$, and $\rho \in(0,1)$,

$$
\liminf _{n \rightarrow \infty} F_{\sum_{k=0}^{n+1} m_{k}+j_{n+1}, \sum_{k=0}^{n+1} m_{k}+i_{n+1}}(\phi(t)) \geq F_{x_{0}, \bar{x}_{1}}(\phi(\infty))=1, \quad \forall t \in \mathbf{R}_{+},
$$

so that the limit below exists being unity for any given integers satisfying $1<i_{n}<j_{n} \leq m_{n+1}$, $\forall n \in \mathbf{Z}_{0+}$ :

$$
\begin{aligned}
& \lim _{n \rightarrow \infty} F_{\sum_{k=0}^{n+1} m_{k}+j_{n+1}{ }^{, x} \sum_{k=0}^{n+1} m_{k}+i_{n+1}}(\phi(t)) \\
& =\lim _{n \rightarrow \infty} \lim _{z \rightarrow 0^{+}} F_{\sum_{\sum_{k=0}^{n+1} m_{k}+j_{n+1}, x} \sum_{k=0}^{n+1} m_{k}+i_{n+1}}(\phi(z))=1, \quad \forall t \in \mathbf{R}_{+},
\end{aligned}
$$

and

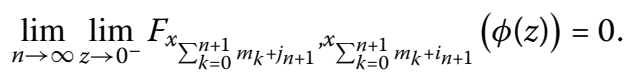

As a result, from the first property of $(X, F, \Delta)$ in (2.1) and since $\phi(0)=0, \phi: \mathbf{R} \rightarrow \mathbf{R}_{0+}$ is left continuous and strictly increasing:

$$
\lim _{n \rightarrow \infty} F_{x_{n}, x_{n+1}}(\phi(t))=\lim _{n \rightarrow \infty} \lim _{z \rightarrow 0^{+}} F_{x_{n}, x_{n+1}}(\phi(z))=\lim _{n \rightarrow \infty} F_{x_{n}, x_{n+1}}\left(0^{+}\right)=1, \quad \forall t \in \mathbf{R}_{+},
$$

and

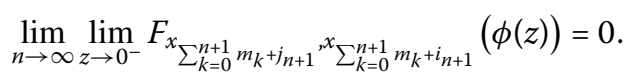

This implies that $\left\{x_{n}\right\}$ converges, necessarily to the same limit $x=\bar{x}=0$ as that of its subsequence $\left\{\bar{x}_{n}\right\}$ irrespective of the initial condition. Since $\left(X, F, \Delta_{M}\right)$ is complete, $\left\{x_{n}\right\}$ is a Cauchy sequence.

The next result does not require that $T_{n} 0=0, \forall n \in \mathbf{Z}_{0+}$ while it invokes the assumptions that $\left\{T_{n}\right\}_{\rightarrow} T^{*}$ and that $T^{*}$ satisfies a $\phi$-contractive condition like (3.18a).

Theorem 3.3 Assume that $\left\{T_{n}\right\}_{\rightarrow} T^{*}$, codomain $T_{n} \subseteq$ domain $T^{*}$ and codomain $T^{*} \subseteq$ domain $T_{n}, \forall n \in \mathbf{Z}_{0+}$, and that all the remaining conditions of all the properties of Theorem 3.2 hold. Then Theorem 3.2 still holds with a unique fixed point of the limit selfmapping $T^{*}$ on $X$. 
Assume, in addition, that if $z_{n} \in \operatorname{Fix}\left(T_{n}\right) ; \forall n \in \mathbf{Z}_{0_{+}}$, and $\operatorname{Fix}\left(T^{*}\right)=x$, then $F_{z_{n}, x}\left(0^{+}\right) \rightarrow 1$ as $n \rightarrow \infty$.

Proof The proof that all the solution sequences built from any given initial condition converge and are Cauchy sequences is the same as that for Theorem 3.2. First note that $\left\{T_{n}\right\}_{\rightarrow}^{\rightarrow} T^{*}$ implies $\left\{T_{n}^{2}\right\}_{\rightarrow} \rightarrow T^{* 2},\left\{T_{n} T\right\}_{\rightarrow} T^{* 2}$ and $\left\{T T_{n}\right\}_{\rightarrow} \rightarrow T^{* 2}$. The proof is as follows. Since $\left\{T_{n}\right\}_{\rightarrow}^{\rightarrow} T^{*}$ we have $\left\{T_{n} x-T^{*} x\right\} \rightarrow 0$ and $\left\{T_{n}^{2} x-T_{n} T^{*} x\right\} \rightarrow 0$ and $\left\{T^{*} T_{n} x-T^{*^{2}} x\right\} \rightarrow 0$, since codomain $T_{n} \subseteq$ domain $T^{*}$ and codomain $T^{*} \subseteq$ domain $T_{n}, \forall n \in \mathbf{Z}_{0+}$, so that $\left\{T_{n}^{2}\right\}_{\rightarrow}\left\{T_{n} T^{*}\right\}$ and $\left\{T^{*} T_{n}\right\}_{\rightarrow}\left\{T^{*^{2}}\right\}, \forall n \in \mathbf{Z}_{0+}$. Via recursion, we get $\left\{T_{n}^{p-1} T^{*}\right\},\left\{T^{*} T_{n}^{p-1}\right\}_{\rightarrow}\left\{T^{*^{p}}\right\}, \forall n \in \mathbf{Z}_{0+}$.

Now, assume that a sequence solution $\left\{x_{n}\right\} \rightarrow x \neq T^{*} x$ so that $F_{x, T^{*} x}\left(0^{+}\right)<1$. Then, for any given $\varepsilon \in \mathbf{R}^{+}$and $\lambda \in(0,1)$,

$$
\begin{aligned}
& F_{x, T^{*} x}(\phi(\varepsilon)) \\
& \geq \Delta_{M}\left(F_{x, x_{n}}(\phi(\varepsilon) / 2), F_{x_{n}, T^{*} x}(\phi(\varepsilon) / 2)\right) \\
& \geq \Delta_{M}\left(F_{x, x_{n}}(\phi(\varepsilon) / 2), \Delta_{M}\left(F_{x_{n}, x_{n+1}}(\phi(\varepsilon) / 4), F_{T^{*} x, T_{n} x_{n}}(\phi(\varepsilon) / 4)\right)\right) \\
& \geq \Delta_{M}\left(F_{x, x_{n}}(\phi(\varepsilon) / 2), \Delta_{M}\left(F_{x_{n}, x_{n+1}}(\phi(\varepsilon) / 4),\right.\right. \\
&\left.\left.\Delta_{M}\left(F_{T_{n} x_{n}, T_{n} x}(\phi(\varepsilon) / 8), F_{T^{*} x, T_{n} x}(\phi(\varepsilon) / 8)\right)\right)\right) \\
&> \Delta_{M}\left(1-\lambda, \Delta_{M}\left(1-\lambda, \Delta_{M}(1-\lambda, 1-\lambda)\right)\right)=1-\lambda, \quad \forall n \geq n_{0},
\end{aligned}
$$

for some nonnegative integers $n_{0 i}=n_{0 i}(\phi(\varepsilon) / 4, \lambda), i=1,2,3$, and $n_{0} \geq \max \left(n_{01}, n_{02}, n_{03}\right)$ which exist such that $\min \left(F_{x, x_{n}}(\phi(\varepsilon) / 8), F_{x_{n}, x_{n+1}}(\phi(\varepsilon) / 8), F_{T_{n} x_{n}, T_{n} x}(\phi(\varepsilon) / 8), F_{T^{*} x, T_{n} x}(\phi(\varepsilon) / 8)\right)>$ $1-\lambda, \forall n \geq n_{0}$ since $\left\{x_{n}\right\} \rightarrow x$ and $\left\{T_{n}\right\}_{\rightarrow} T^{*}$, so that $\left\{T_{n} x-T_{n} x\right\} \rightarrow 0$ and $\left\{T_{n} x-T^{*} x\right\} \rightarrow 0$. Since $x \neq T^{*} x, \phi(0)=0, \phi: \mathbf{R} \rightarrow \mathbf{R}_{0+}$ is left continuous and strictly increasing and since $\lambda \in(0,1)$ is arbitrary, the limits below exist from the first property of the PM space $(X, F, \Delta)$ in (2.1):

$$
\lim _{\varepsilon \rightarrow 0^{+}} F_{x, T^{*} x}(\phi(\varepsilon))=F_{x, T^{*} x}\left(0^{+}\right)=1 ; \quad \lim _{\varepsilon \rightarrow 0^{-}} F_{x, T^{*} x}(\phi(\varepsilon))=F_{x, T^{*} x}\left(0^{-}\right)=0
$$

so that if $\left\{T_{n}\right\}_{\rightarrow} \rightarrow T^{*}$ and $\left\{T_{n} x_{n}\right\} \rightarrow x$ then $x=T^{*} x$, that is, the limiting point is a fixed point of the limit self-mapping. Thus, the limit of any solution sequence is a fixed point of the limit self-mapping. It is now proved by contradiction that the fixed point is unique. Assume that for an initial condition $x_{0}$, the solution sequence $\left\{x_{n}\right\} \rightarrow x$ and for some initial condition $y_{0} \neq x_{0}$, the solution sequence $\left\{y_{n}\right\} \rightarrow y=T^{*} y \neq x$ and that $x \neq y$. Then, from the $\phi$-contractive condition (3.18a) and since $\phi: \mathbf{R} \rightarrow \mathbf{R}_{0+}$ is left continuous and strictly increasing and since the probability distribution function is left continuous and non-decreasing for any given $\varepsilon \in \mathbf{R}_{+}$and $\lambda \in(0,1)$ and $\varepsilon_{1}=\varepsilon_{1}(\varepsilon)=\min \left(r \in \mathbf{R}_{+}: \varepsilon=\phi\left(\varepsilon_{1}^{+}\right)\right)$:

$$
\begin{aligned}
F_{T^{*} x, T^{*} y}(\varepsilon) & \geq \Delta_{M}\left(F_{T^{*} x, T_{n} \bar{x}_{n}}(\varepsilon / 2), \Delta_{M}\left(F_{T_{n} \bar{x}_{n}, T_{n} \bar{y}_{n}}(\varepsilon / 4), F_{T_{n} \bar{y}_{n}, T^{*} y}(\varepsilon / 4)\right)\right) \\
& \geq \Delta_{M}\left(F_{T^{*} x, T_{n} \bar{x}_{n}}(\varepsilon / 4), \Delta_{M}\left(F_{T_{n} \bar{x}_{n}, T_{n} \bar{y}_{n}}\left(\phi\left(\varepsilon_{1}^{+}\right) / 4\right), F_{T_{n} \bar{y}_{n}, T^{*} y}(\varepsilon / 4)\right)\right) \\
& \geq \Delta_{M}\left(F_{T^{*} x, T_{n} \bar{x}_{n}}(\varepsilon / 4), \Delta_{M}\left(F_{x, y}\left(\phi\left(\rho^{-n} \rho_{n}^{-1} \varepsilon_{1}^{+}\right) / 4\right), F_{T_{n} \bar{y}_{n}, T^{*} y}(\varepsilon / 4)\right)\right)
\end{aligned}
$$

for subsequences $\left\{\bar{x}_{n}\right\} \subset\left\{x_{k}\right\} \subset X$ and $\left\{\bar{y}_{n}\right\} \subset\left\{y_{k}\right\} \subset X$ built with the initial conditions $\bar{x}_{0}=x_{0}=x=T^{*} x$, since $x \in \operatorname{Fix}\left(T^{*}\right)$, and $\bar{y}_{0}=y_{0}=y=T^{*} y$, since $y \in \operatorname{Fix}\left(T^{*}\right)$, respectively. 
Also,

$$
\begin{aligned}
& F_{T^{*} x, T_{n} \bar{x}_{n}}(\varepsilon / 4) \geq \Delta_{M}\left(F_{T^{*} x, T_{n} x}(\varepsilon / 8), F_{T_{n} \bar{x}_{n}, T_{n} x}(\varepsilon / 8)\right)>1-\lambda, \\
& F_{T^{*} y, T_{n} \bar{y}_{n}}(\varepsilon / 4) \geq \Delta_{M}\left(F_{T^{*} y, T_{n} y}(\varepsilon / 8), F_{T_{n} \bar{y}_{n}, T_{n} y}(\varepsilon / 8)\right)>1-\lambda,
\end{aligned}
$$

for $n \geq \bar{n}_{01}=\max \left(n_{00 i} \in \mathbf{Z}_{0+}: i \in \overline{4}\right)$ with each of the integers $n_{00 i}$ being large enough to satisfy the requirement that the corresponding one of the four terms being arguments of $\Delta_{M}(\cdot, \cdot)$ exceeds $1-\lambda$. Such four integers exist since $\left\{T^{*} x-T_{n} x\right\} \rightarrow 0,\left\{T_{n} \bar{x}_{n}-T_{n} x\right\} \rightarrow 0$, $\left\{T^{*} y-T_{n} y\right\} \rightarrow 0$ and $\left\{T_{n} \bar{y}_{n}-T_{n} y\right\} \rightarrow 0$ (since $\left\{T_{n}\right\}_{\rightarrow} T^{*},\left\{T_{n} \bar{x}_{n}\right\} \rightarrow x=T^{*} x$ and $\left\{T_{n} \bar{y}_{n}\right\} \rightarrow$ $y=T^{*} y$ ), we have $F_{T^{*} x, T_{n} x}(t) \rightarrow 1$ as $n \rightarrow \infty, F_{T_{n} \bar{x}_{n}, T_{n} x}(t) \rightarrow 1$ as $n \rightarrow \infty, F_{T^{*} y, T_{n} y}(t) \rightarrow 1$ as $n \rightarrow \infty$ and $F_{T_{n} \bar{y}_{n}, T_{n} y}(\varepsilon / 8) \rightarrow 1$ as $n \rightarrow \infty$ for all $t \in \mathbf{R}_{+}$from the first property of the Menger PM space $(X, F, \Delta)$ in (2.1). There is also $n_{005} \in \mathbf{Z}_{0+}$ such that $F_{x, y}\left(\phi\left(\rho^{-n} \rho_{n}^{-1} \varepsilon_{1}^{+}\right) / 4\right)>$ $1-\lambda$ for $n \geq n_{005}$, since there exists $\lim _{n \rightarrow \infty} F_{x, y}\left(\phi\left(\rho^{-n} \rho_{n}^{-1} \varepsilon_{1}^{+}\right) / 4\right)=F_{x, y}(\infty)=1$ from the $\phi$ contractive condition. Now, from (3.28) into (3.27), and taking $n \geq \bar{n}_{0 a}=\max \left(\bar{n}_{0}, n_{005}\right)$ in the last right-hand side term, one gets that $F_{T^{*} x, T^{*} y}(t)=F_{T^{*} x, T^{*} y}\left(0^{+}\right)=1, \forall t \in \mathbf{R}_{+}$since for any given arbitrary $\varepsilon \in \mathbf{R}_{+}$and $\lambda \in(0,1), F_{T^{*}, T^{*} y}(\varepsilon)>1-\lambda$. Thus, $x=T^{*} x=T^{*} y=y$ from the first property of the Menger PM space $(X, F, \Delta)$ in (2.1). Then $\operatorname{Fix}\left(T^{*}\right)=\{x\}$.

Finally, it is proved that if $\left\{T_{n}\right\}_{\rightarrow}^{\rightarrow} T^{*}, z_{n} \in \operatorname{Fix}\left(T_{n}\right), \forall n \in \mathbf{Z}_{0+}$ and $\operatorname{Fix}\left(T^{*}\right)=\{x\} ; \forall n \in \mathbf{Z}_{0+}$, then $F_{z_{n}, x}\left(0^{+}\right) \rightarrow 1$ as $n \rightarrow \infty$. Assume that $F_{z_{n}, x}\left(0^{+}\right) \rightarrow 1$ as $n \rightarrow \infty$ is untrue. Since $\left\{T_{n}\right\}_{\rightarrow} \rightarrow T^{*}$ and the $\phi$-contractive condition (3.18a), together with (3.18b), hold, one gets that if $z_{n} \in \operatorname{Fix}\left(T_{n}\right)$, then $z_{n}=T_{n} z_{n}=T_{n}^{m} z_{n}$ and $x=T^{*} x=T^{* m} x$ for each given $\varepsilon \in \mathbf{R}_{+}$, $\forall n, m \in \mathbf{Z}_{0+}$, and there is $\lambda_{1}=\lambda_{1}(\varepsilon) \in(0,1)$ such that

$$
\begin{aligned}
1-\lambda_{1} & \geq F_{z_{n}, x}(\varepsilon) \geq \Delta_{M}\left(F_{T_{n} z_{n}, T_{n} x}(\varepsilon / 2), F_{T_{n} x, T^{*} x}(\varepsilon / 2)\right) \\
& >\min \left(F_{T_{n} z_{n}, T_{n} x}(\varepsilon / 2), 1-\lambda\right) ; \quad \forall n \geq N_{0}
\end{aligned}
$$

for any given $\varepsilon \in \mathbf{R}_{+}$and $\lambda \in(0,1)$ and some $N_{0}=N_{0}(\varepsilon, \lambda) \in \mathbf{Z}_{0+}$ since $\left\{T_{n} x-T^{*} x\right\} \rightarrow 0$. The proof is split into three cases: a) If $F_{T_{n} z_{n}, T_{n} x}(\varepsilon / 2) \geq 1-\lambda, \forall n \geq N_{0}$, then $\lambda_{1}<\lambda$. Since $\lambda \in$ $(0,1)$ is arbitrary, it suffices to take $\lambda \in\left(0, \lambda_{1}\right]$ to get a contradiction so that $F_{z_{n}, x}\left(0^{+}\right) \rightarrow 1$ as $n \rightarrow \infty$. b) Assume that there is a set of nonnegative integers $\left\{\hat{n}_{k}\right\}$ fulfilling $\hat{n}_{k}\left(\in \mathbf{Z}_{0+}\right) \geq N_{0}$ such that $F_{\hat{n}_{k}} z_{\hat{n}}, T_{\hat{n}_{k}} x(\varepsilon / 2)<\min \left(1-\lambda, 1-\lambda_{1}\right)$. If the set is finite, it suffices to use the above arguments for the case a by replacing $N_{0} \rightarrow \bar{N}_{0}=\left\{\max z \in \mathbf{Z}_{0+}: z \in\left\{\hat{n}_{k}\right\}\right\}$ to get the same conclusion as above. c) Assume that $\left\{\hat{n}_{k}\right\}$, satisfying $\hat{n}_{k}\left(\in \mathbf{Z}_{0+}\right) \geq N_{0}$, is a strictly increasing sequence.

Set $\hat{n}_{k_{*}}=\max \left(z\left(\in \mathbf{Z}_{0_{+}}\right) \leq \hat{n}_{k}+1: x_{\hat{n}_{k_{*}}} \in\left\{\bar{x}_{n_{k}}\right\} \subset\left\{x_{n}\right\}\right)$, the closest, but not larger, integer to $\hat{n}_{k}+1$ such that $x_{\hat{n}_{k *}}$ belongs to the subsequence $\left\{\bar{x}_{n_{k}}\right\} \subset\left\{x_{n}\right\}$ whose elements satisfy the $\phi$-contractive condition. Then one gets for $\varepsilon_{1}=\min \left(r \in \mathbf{R}_{+}: \varepsilon=2 \phi\left(\varepsilon_{1}^{+}\right)\right)$some sequence of real constants $\left\{K_{\hat{n}_{k}}\right\} \subset \mathbf{R}_{+}$, with $0<K_{\hat{n}_{k}} \leq K<\infty, \forall k \in \mathbf{Z}_{0_{+}}$, and some strictly increasing sequence of integers $\left\{\ell_{n_{k}}\right\} \subset \mathbf{Z}_{0+}$ :

$$
\begin{aligned}
& \min \left(1-\lambda, 1-\lambda_{1}\right) \\
& \quad>F_{T_{\hat{n}_{k}} z_{\hat{n}_{k}}, T_{\hat{n}_{k}} x}(\varepsilon / 2) \geq F_{T_{\hat{n}_{k}} z_{\hat{n}_{k}}, T_{\hat{n}_{k}} x}\left(\phi\left(\varepsilon_{1}^{+}\right)\right) \\
& \quad \geq F_{T_{\hat{n}_{k *}} z_{\hat{n}_{k *}}, T_{\hat{n}_{k *}} x}\left(\phi\left(K_{\hat{n}_{k}}^{-1} \varepsilon_{1}^{+}\right)\right) \geq F_{T_{\hat{n}_{0}} z_{\hat{n}_{0 *}}, T_{\hat{n}_{0 *}} x}\left(\phi \left(\rho^{\left.\left.-\ell_{\hat{n}_{k *}} K^{-1} \varepsilon_{1}^{+}\right)\right),}\right.\right.
\end{aligned}
$$


which leads to the following contradiction:

$$
\begin{aligned}
& \min \left(1-\lambda, 1-\lambda_{1}\right) \\
& >\lim \sup _{k \rightarrow \infty} F_{T_{\hat{n}_{0 *}} z_{\hat{n}_{0 *}}, T_{\hat{n}_{0 *}} x}\left(\phi\left(\rho^{-\ell_{\hat{n}_{k}}} K^{-1} \varepsilon_{1}^{+}\right)\right) \\
& =\lim _{k \rightarrow \infty} F_{T_{\hat{n}_{0 *}} z_{\hat{n}_{0 *}}, T_{\hat{n}_{0 *}} x}\left(\phi\left(\rho^{-\ell_{\hat{n}_{k}}} K^{-1} \varepsilon_{1}^{+}\right)\right)=F_{T_{\hat{n}_{0 *}} z_{\hat{n}_{0 *}}, T_{\hat{n}_{0 *}}{ }^{x}}(\infty)=1
\end{aligned}
$$

and $F_{z_{n}, x}\left(0^{+}\right) \rightarrow 1$ as $n \rightarrow \infty$.

The following result reformulates some results of Theorem 3.1 without requiring that the iterative scheme is $x_{n+1}=\sum_{i=1}^{n} \alpha_{i}^{(n)} T^{k} x_{0}, x_{0} \in X$, that $T_{n} 0=0, \forall n \in \mathbf{Z}_{0+}$, that $X$ is a vector space, and that the self-mappings of the sequence $\left\{T_{n}\right\}$ satisfy a probabilistic $\phi$ contractive condition. Instead, it is required that we have uniform convergence of $\left\{T_{n}\right\}$ to a $\phi$-contractive limit self-mapping.

Theorem 3.4 Consider a complete Menger space $\left(X, F, \Delta_{M}\right)$, endowed with the minimum triangular norm $\Delta_{M}$ and the iterative scheme (3.1).

Assume also that the self-mappings $T_{n}: X \rightarrow X, \forall n \in \mathbf{Z}_{0+}$ have fixed points $F_{T_{n}}=\left\{x_{n}^{*}\right\}$, $\forall n \in \mathbf{Z}_{0+}$, that $\left\{T_{n}\right\}_{\rightarrow}^{\rightarrow} T^{*}$ with $T^{*}: X \rightarrow X$ having a fixed point $F_{T^{*}}=\left\{x^{*}\right\}$ and being a $\phi$ contraction which satisfies

$$
F_{T^{*} z_{n+1}, T^{*} z_{n}}(\phi(t)) \geq F_{z_{n+1}, z_{n}}\left(\phi\left(\rho^{-1} t\right)\right), \quad \forall t \in \mathbf{R}_{+}, \forall n \in \mathbf{Z}_{0+}
$$

for some $\Phi$-function $\phi: \mathbf{R} \rightarrow \mathbf{R}_{0+}$ and some real constant $\rho \in(0,1)$. Then the following properties hold:

(i) There exist the limits $\lim _{n \rightarrow \infty} F_{x_{n}, x_{n+1}}(t)=1$ and $\lim _{n \rightarrow \infty} F_{z_{n}, z_{n+1}}(t)=1, \forall t \in \mathbf{R}_{+}$for any given initial points $z_{0}, x_{0} \in X$ of the iterative schemes $x_{n+1}=T_{n} x_{n}$ and $z_{n+1}=T^{*} z_{n}$, generated from (3.1), and $\left\{z_{n}\right\}$ is a Cauchy sequence for any given $z_{0} \in X$, and bounded, and convergent to $z *$. Furthermore, $\left\{x_{n}^{*}\right\} \rightarrow x^{*}$ and $\left\{T^{* n} x\right\} \rightarrow x^{*}$ for any $x \in X$.

(ii) $\left\{T_{n}^{n} x_{n}\right\} \rightarrow x^{*},\left\{T_{n}^{n} x_{n}-x_{n}^{*}\right\} \rightarrow 0,\left\{T_{n}^{n} x_{n}\right\} \rightarrow x^{*}$, and $\left\{x_{n}\right\}$ is a Cauchy sequence, and bounded, for any given initial point $x_{0} \in X$.

Proof One gets from (3.31)

$$
\begin{aligned}
F_{z_{n}, z_{n-1}}(\phi(t)) & \geq F_{z_{n-1}, z_{n-2}}\left(\phi\left(\rho^{-1} t\right)\right) \geq F_{z_{n-2}, z_{n-3}}\left(\phi\left(\rho^{-2} t\right)\right) \\
& \geq \cdots \geq F_{z_{1}, z_{0}}\left(\phi\left(\rho^{-n+1} t\right)\right), \quad \forall n \in \mathbf{Z}_{0+}, \forall t \in \mathbf{R}_{+} .
\end{aligned}
$$

Since there exists $\lim _{n \rightarrow \infty} F_{T^{*} z_{0}, z_{0}}\left(\phi\left(\rho^{-n+1} t\right)\right)=F_{T^{*} z_{0}, z_{0}}\left(\lim _{n \rightarrow \infty} \phi\left(\rho^{-n+1} t\right)\right)=$ $F_{z_{0}, T^{*} z_{0}}(\infty)=1, \forall t \in \mathbf{R}_{+} \lim _{n \rightarrow \infty} F_{T^{* n+1} z_{0}, T^{* n} z_{0}}(\phi(t))=1, \forall t \in \mathbf{R}_{+}$. Since $\phi: \mathbf{R}_{0_{+}} \rightarrow \mathbf{R}_{0+}$ is a $\Phi$-function, $\lim _{n \rightarrow \infty} F_{T^{* n+1} z_{0}, T^{* n} z_{0}}\left(\phi\left(0^{+}\right)\right)=\lim _{n \rightarrow \infty} F_{T^{* n+1} z_{0}, T^{* n} z_{0}}\left(0^{+}\right)=1$ and

$$
\begin{aligned}
\lim _{n \rightarrow \infty} F_{T^{* n+1} z_{0}, T^{* n} z_{0}}(t) & =\lim _{n \rightarrow \infty} F_{T^{* n+1} z_{0}, T^{* n} z_{0}}\left(t^{-}\right) \\
& =F_{z_{0}, T^{*} z_{0}}(\infty)=1 \quad \text { for any given } z_{0} \in X .
\end{aligned}
$$


It then turns out that, since $\left(X, F, \Delta_{M}\right)$ is a Menger PM space, for any given $\varepsilon \in \mathbf{R}_{+}$and $\lambda \in$ $(0,1)$, there is $n_{0}=n_{0}(\varepsilon, \lambda)$ such that $F_{T^{* n+1} z_{0}, T^{* n} z_{0}}(\varepsilon) \geq 1-\lambda$ for any $n>n_{0}$ so that $\left\{T^{* n} z_{0}\right\}$ is a Cauchy sequence, which is bounded, and since $\left(X, F, \Delta_{M}\right)$ is complete, it converges to some $z \in X$. Assume that $z \neq x^{*}$ so that $F_{x^{*}, z}\left(0^{+}\right)<1$. Since $F_{z *, T^{*} x^{*}}\left(0^{+}\right)=1$, since $F_{T^{*}}=\left\{x^{*}\right\}$, one gets

$$
1>F_{x^{*}, z}\left(0^{+}\right)=F_{T^{* n} x^{*}, z}\left(0^{+}\right) \geq \Delta_{M}\left(F_{T^{* n} x^{*}, T^{* n} z_{0}}\left(0^{+} / 2\right), F_{z, T^{* n} z_{0}}\left(0^{+} / 2\right)\right)
$$

and, since $\left\{T^{* n} z_{0}\right\} \rightarrow z, \lim _{n \rightarrow \infty} F_{z, T^{* n} z_{0}}\left(0^{+} / 2\right)=\lim _{n \rightarrow \infty} F_{z, T^{* n} z_{0}}\left(0^{+} / 2\right)=1$, so that one has for a sufficiently large integer $n_{01}$ and all integers $n>n_{01}$

$$
\begin{aligned}
1 & >F_{x^{*}, z}(\phi(t))=F_{T^{* n} x^{*}, z}(\phi(t)) \geq \Delta_{M}\left(F_{T^{* n} x^{*}, T^{* n} z_{0}}(\phi(t) / 2), F_{z, T^{* n} z_{0}}(\phi(t) / 2)\right) \\
& \geq F_{T^{* n} x^{*}, T^{* n} z_{0}}(\phi(t) / 2) \geq F_{x^{*}, z_{0}}\left(\rho^{-n} \phi\left(t_{1}\right)\right), \quad \forall t \in \mathbf{R}_{+},
\end{aligned}
$$

where $t_{1} \in \mathbf{R}_{+}$is such that $\phi\left(t_{1}\right)=\lim _{x \rightarrow t_{1}^{-}} \phi(x)=\phi(t) / 2=(1 / 2) \lim _{x \rightarrow t^{-}} \phi(x)$ for any given $t \in \mathbf{R}_{+}$with $t_{1}<t$ for $t \in \mathbf{R}_{+}$, since $\phi: \mathbf{R}_{0_{+}} \rightarrow \mathbf{R}_{0_{+}}$is a $\Phi$-function, it is strictly increasing and left continuous except at $t=0$ where it is zero and continuous. Taking limits as $n \rightarrow \infty$, one gets the contradiction $1>\lim _{n \rightarrow \infty} F_{x^{*}, z_{0}}\left(\phi\left(\rho^{-n} t_{1}\right)\right)=F_{z *, z_{0}}(\infty)=1$.

Then $F_{x^{*}, z}(t)=1, \forall t \in \mathbf{R}_{+}$, and from the first property of the Menger PM space $(X, F, \Delta)$, $x^{*}=z$ irrespective of the initial point $z_{0} \in X$ of the iteration $z_{n+1}=T^{*} z_{n}, \forall n \in \mathbf{Z}_{0+}$. It has been proved that $\left\{z_{n}\right\} \rightarrow x^{*}$ irrespective of the initial condition $z_{0}$. It is now proved that $\left\{x_{n}^{*}\right\} \rightarrow x^{*}$. Proceed by contradiction by assuming that this convergence fails. Then there exists some $\lambda_{0} \in(0,1) \cap \mathbf{R}$ such that, for any $n \in \mathbf{Z}_{0_{+}}$, one gets for an existing $t_{1}=t_{1}(t) \in \mathbf{R}_{+}$ for any given $t \in \mathbf{R}_{+}$, defined by $\phi\left(t_{1}\right)=\phi(t) / 2$, the following inequalities:

$$
\begin{aligned}
1-\lambda_{0} & >F_{T_{n} x_{n}^{*}, T^{* n} x^{*}}(\phi(t)) \geq \Delta_{M}\left(F_{T_{n} x_{n}^{*}, T^{* n} x_{n}^{*}}(\phi(t) / 2), F_{T^{* n} x_{n}^{*}, T^{* n} x^{*}}(\phi(t) / 2)\right) \\
& \geq \Delta_{M}\left(F_{T_{n} x_{n}^{*}, T^{* n} x_{n}^{*}}(\phi(t) / 2), F_{x_{n}^{*}, x^{*}}\left(\phi\left(\rho^{-n} t_{1}\right)\right)\right),
\end{aligned}
$$

since $\phi: \mathbf{R}_{0+} \rightarrow \mathbf{R}_{0+}$ is a $\Phi$-function and $\lim _{n \rightarrow \infty} F_{T^{* n} x_{n}^{*}, T^{* n} x^{*}}\left(\phi\left(\rho^{-n} t_{1}\right)\right)=1, \forall t_{1} \in \mathbf{R}_{+}$, since $T^{*}$ is a $\phi$-contraction on $X$. Then there exists $n_{1}=n_{1}\left(t_{1}(t), \lambda\right) \in \mathbf{Z}_{0+}$ for any given $t \in \mathbf{R}_{+}$ and $\lambda \in(0,1)$ such that $F_{x_{n}^{*}, x^{*}}\left(\phi\left(\rho^{-n} t_{1}\right)\right) \geq 1-\lambda$ for all integers $n>n_{1}$. Then, from (3.35),

$$
\begin{aligned}
1-\lambda_{0} & >\Delta_{M}\left(F_{T_{n} x_{n}^{*}, T^{* n} x_{n}^{*}}(\phi(t) / 2), F_{x_{n}^{*}, x^{*}}\left(\phi\left(\rho^{-n} t_{1}\right)\right)\right) \\
& \geq \Delta_{M}\left(F_{T_{n} x_{n}^{*}, T^{* n} x_{n}^{*}}(\phi(t) / 2), 1-\lambda\right)
\end{aligned}
$$

for all integers $n>n_{1}$ and, since $T^{* n} x^{*}=T^{*} x^{*}=x^{*}$ and, again, since $T^{*}$ is a $\phi$-contraction on $X$ and $\lim _{n \rightarrow \infty} F_{x_{n}^{*}, x^{*}} \phi\left(\rho^{-n} t_{1}\right)=1$, there exists $n_{2}=n_{2}(t, \lambda)\left(>n_{1}\right) \in \mathbf{Z}_{0+}$ such that $F_{x_{n}^{*}, x^{*}} \phi\left(\rho^{-n} t_{1}\right) \geq 1-\lambda$, for all integers $n>n_{2}$. Thus, for all integers $n \geq \max \left(n_{1}, n_{2}\right)$, the following contradiction arises from (3.36) for the choice $\lambda\left(>\lambda_{0}\right) \in(0,1)$ for the given $\lambda_{0} \in \mathbf{R}_{+}$:

$$
1-\lambda_{0}>\Delta_{M}\left(\Delta_{M}\left(F_{x_{n}^{*}, x^{*}} \phi\left(\rho^{-n} t_{1}\right), F_{T^{*} x^{*}, T_{n} x_{n}^{*}}(\phi(t) / 2)\right), 1-\lambda\right) \geq 1-\lambda .
$$

It has been proved that $\left\{x_{n}^{*}\right\} \rightarrow x^{*}$. It is now proved that $\left\{T^{* n} x\right\} \rightarrow x^{*}$ for any $x \in X$. As in the preceding recursions with the $\phi$-contractive condition, one easily gets

$$
F_{T^{* n} x, T^{* n} x^{*}}(\phi(t)) \geq F_{x, x^{*}}\left(\phi\left(\rho^{-n} t\right)\right) ; \quad \forall n \in \mathbf{Z}_{0+} .
$$


Then

$$
\lim _{n \rightarrow \infty} F_{T^{* n} x, T^{* n} x^{*}}\left(\phi\left(0^{+}\right)\right)=F_{x, z *}(\infty)=1, \quad F_{T^{* n} x, T^{* n} x^{*}}(\varepsilon) \geq 1-\lambda
$$

for any given $\varepsilon \in \mathbf{R}_{+}$, some $t \in \mathbf{R}_{+}$satisfying uniquely $\phi(t)=\varepsilon$ (from the properties of the $\Phi$-functions) and all integers $n>n^{*}$ and some $n^{*}=n^{*}(\varepsilon, \lambda) \in \mathbf{Z}_{0+}$. Property (i) has been proved.

It is now proved that $\left\{T_{n}^{n} x_{n}-x_{n}^{*}\right\} \rightarrow 0$ and $\left\{T_{n}^{n} x_{n}\right\} \rightarrow z^{*}$. Assume, on the contrary, that $\left\{T_{n}^{n} x_{n}-x_{n}^{*}\right\}$ does not converge to zero, so that $\limsup _{n \rightarrow \infty} F_{T_{n}^{n} x_{n}, *}(t)<1, \forall t \in \mathbf{R}_{+}$. Then there exists a positive real subsequence $\left\{\lambda_{0}\left(n_{k}\right)\right\} \subset(0,1]$ that is defined by $\lambda_{0}\left(n_{k}\right)=$ $1-F_{T_{n_{k}}^{n} x_{n_{k}}, x_{n_{k}}^{*}}\left(t_{1}\right)$, where $\left\{n_{k}\right\} \subset \mathbf{Z}_{0+}$ is a strictly increasing subsequence of the set of nonnegative integers, such that for an existing unique $t_{1}=\phi(t) \in \mathbf{R}_{+}$, for any given $t \in \mathbf{R}_{+}$, which follows as in the repeated arguments used in the proof of Property (i):

$$
\begin{aligned}
1 & >1-\lambda_{0}\left(n_{k}\right) \\
& =F_{T_{n_{k}}^{n} x_{n_{k}}, x_{n_{k}}^{*}}\left(t_{1}\right) \geq \Delta_{M}\left(F_{x_{n_{k}}^{*}, x^{*}}\left(t_{1} / 2\right), F_{x^{*}, T_{n_{k}}^{n} x_{n_{k}}}\left(t_{1} / 2\right)\right) \geq \min \left(1-\lambda, F_{x^{*}, T_{n_{k}}^{n} x_{n_{k}}}\left(t_{1} / 2\right)\right) \\
& =F_{x^{*}, T_{n_{k}}^{n_{k}} x_{n_{k}}}\left(t_{1} / 2\right) \geq \Delta_{M}\left(F_{x^{*}, T^{* n} x_{n_{k}}}\left(t_{1} / 4\right), F_{T^{* n} n_{n_{k}}, T_{n_{k}}^{n} x_{n_{k}}}\left(t_{1} / 4\right)\right) \\
& \geq \min \left(1-\lambda, F_{T^{* n} x_{n_{k}}, T_{n_{k}}^{n} x_{n_{k}}}\left(t_{1} / 4\right)\right)=1-\lambda
\end{aligned}
$$

for all $n\left(\in \mathbf{Z}_{0+}\right)>n_{\alpha}=n_{\alpha}\left(t_{1}, \lambda\right)$, some $n_{\alpha}=n_{\alpha}\left(t_{1}, \lambda\right) \in \mathbf{Z}_{0+}$ and some $\left\{\lambda_{0}\left(n_{k}\right)\right\} \subset(0,1]$ such that

$$
1-\lambda_{0}\left(n_{k}\right) \geq \min \left(F_{x_{n_{k}}^{*}, z^{*}}\left(t_{1} / 2\right), F_{z *, T^{* n} x_{n_{k}}}\left(t_{1} / 4\right), F_{T^{* n} n_{n_{k}}, T_{n_{k}}^{n} x_{n_{k}}}\left(t_{1} / 4\right)\right),
$$

which holds since $\left\{x_{n_{k}}^{*}\right\} \subset\left\{x_{n}^{*}\right\}\left(\rightarrow x^{*}\right), T^{* n} x_{n_{k}}\left(\rightarrow x^{*}\right)$ for any fixed $x_{n_{k}} \subset X$, being any arbitrary element of the solution sequence $\left\{x_{n}\right\}$ generated by (3.1) for any given $x_{0} \in X$. Thus, $F_{x_{n_{k}}^{*}, x^{*}}\left(t_{1} / 2\right) \rightarrow 1, F_{x^{*}, T^{*} n_{k} x_{n_{k}}}\left(t_{1} / 4\right)$, and $F_{T^{* n} x_{n_{k}}, T_{n_{k}}^{n} x_{n_{k}}}\left(t_{1} / 4\right) \rightarrow 1$ as $n, k \rightarrow \infty$ (and then $\left.\left\{n_{k}\right\} \rightarrow \infty\right)$ from Property (i) and the fact that $\left\{T_{n}\right\}_{\rightarrow} T^{*}$. Now, the choice $\lambda \in$ $\left(0, \liminf \lambda_{0}\left(n_{k}\right)_{k \rightarrow \infty}\right)$ contradicts (3.40). As a result, $\left\{T_{n}^{n} x_{n}-x_{n}^{*}\right\} \rightarrow 0$ since all its subsequences have this property of convergence to zero. Finally, the convergence $\left\{T_{n}^{n} x_{n}\right\} \rightarrow x^{*}$ follows under very close arguments from the fact that $\left\{x_{n}^{*}\right\} \rightarrow x^{*}$, from Property (i), $\left\{T_{n}^{n} x_{n}-x_{n}^{*}\right\} \rightarrow 0$ already proved as a part of Property (ii), and the inequality $F_{T_{n}^{n} x_{n}, x^{*}}(\phi(t)) \geq$ $\Delta_{M}\left(F_{T_{n}^{n} x_{n}, x_{n}^{*}}(\phi(t) / 2), F_{x_{n}^{*}, x^{*}}(\phi(t) / 2)\right)$ with both inequality sides having as limit unity as $n \rightarrow \infty$. To prove that $\left\{x_{n}\right\}$ is a Cauchy sequence, first define the composite self-mapping $\hat{T}(n+1, j)=T_{n} \hat{T}(n, j)=T_{n} T_{n-1} \cdots T_{j+1} T_{j}, \forall j, n\left(\in \mathbf{Z}_{0+}\right) \leq n+1$ from $X$ to $X$, subject to $\hat{T}(n, n)=I, \forall n \in \mathbf{Z}_{0+}$, and note that

$$
\begin{aligned}
& x_{n+1}=\hat{T}(n+1, j) x_{j}=\hat{T}(n+1,0) x_{0} ; \\
& \bar{x}_{n+1}(j)=T^{* n-j} x_{j}=T^{* n-j} x_{j} \hat{T}(j, 0) x_{0} ; \\
& z_{n+1}=T^{* n-j} z_{j}=T^{*-j} z_{0}, \quad \forall j, n\left(\in \mathbf{Z}_{0+}\right) \leq n+1, \forall x_{0}, z_{0} \in X .
\end{aligned}
$$

It has been proved in Property (i) that $\left\{z_{n}\right\} \rightarrow x^{*}=T^{*} x^{*}$ for any given $z_{0} \in X$. As a result, $\left\{\bar{x}_{n}(j)-z_{n+1}\right\} \rightarrow 0$ and $\left\{\bar{x}_{n}(j)\right\} \rightarrow x^{*}$ for any $j \in \mathbf{Z}_{0+}$ irrespective of $x_{j}$ and $z_{0}$ being equal 
or distinct and $F_{\bar{x}_{n+1}(j), x^{*}}(\varepsilon) \geq 1-\lambda$ for any given $\lambda \in(0,1)$ and $t, \varepsilon \in \mathbf{R}_{+}$, with $\varepsilon=\phi(t)$, $\forall n\left(\in \mathbf{Z}_{0+}\right)>n_{0 s}=n_{0 s}(\varepsilon, \lambda)$, where $n_{0 s} \in \mathbf{Z}_{0+}$ exists from the convergence property. Now, since $\left\{T_{n}\right\}_{\rightarrow} \rightarrow T^{*}$ then $\{\hat{T}(n+1, j)\} \rightarrow T^{* n-j}$ for all $n(>j)$ as $j \rightarrow \infty$, and

$$
\begin{aligned}
F_{x_{n+1}, x^{*}}(\varepsilon) & \geq \Delta_{M}\left(F_{x_{n+1}, \bar{x}_{n+1}(j)}(\varepsilon / 2), F_{\bar{x}_{n+1}(j), x^{*}}(\varepsilon / 2)\right) \\
& \geq \Delta_{M}\left(F_{\hat{T}(n+1, j) x_{j}, T^{* n-j} x_{j}}(\varepsilon / 2), 1-\lambda\right),
\end{aligned}
$$

and there is $j_{0 s}=j_{0 s}(\varepsilon, \lambda)$ such that for all $n>j>j_{0 s}, F_{\hat{T}(n+1, j) x_{j}, T^{* n-j_{x}}}(\varepsilon / 2) \geq 1-\lambda$. Then

$$
\begin{aligned}
& F_{x_{n+1}, x^{*}}(\varepsilon) \geq \Delta_{M}(1-\lambda, 1-\lambda)=1-\lambda, \\
& F_{x_{n+2}, x_{n+1}}(\varepsilon) \geq \Delta_{M}\left(F_{x_{n+2}, x^{*}}(\varepsilon), F_{x_{n+1}, x^{*}}(\varepsilon)\right)=1-\lambda,
\end{aligned}
$$

$\forall n\left(\in \mathbf{Z}_{0+}\right)>\max \left(n_{0 s}, j_{0 s}+1\right)$. Thus, $\left\{x_{n}\right\} \rightarrow x^{*}$ and it is a Cauchy sequence.

The subsequent result gives close results to those obtained in the above one in the case when $\left\{T_{n}\right\} \rightarrow T^{*}$ (i.e. under point-wise convergence of the self-mappings).

Theorem 3.5 Let $\left(X, F, \Delta_{M}\right)$ be a complete Menger space endowed with the minimum triangular norm $\Delta_{M}$ and consider the iterative scheme (3.1). Assume also that the selfmappings $T_{n}: X \rightarrow X, \forall n \in \mathbf{Z}_{0_{+}}$have unique fixed points $F_{T_{n}}=\left\{x_{n}^{*}\right\}, \forall n \in \mathbf{Z}_{0+}$, that $\left\{T_{n}\right\} \rightarrow T^{*}$ for some $T^{*}: X \rightarrow X$, and that the following $\phi$-contractive condition is satisfied:

$$
F_{T_{n} z_{n+1}, T_{n} z_{n}}(\phi(t)) \geq F_{z_{n+1}, z_{n}}\left(\phi\left(\rho_{n}^{-1} t\right)\right), \quad \forall t \in \mathbf{R}_{+}, \forall n \in \mathbf{Z}_{0+}
$$

for some $\Phi$-function $\phi: \mathbf{R} \rightarrow \mathbf{R}_{0+}$ and some real sequence $\left\{\rho_{n}\right\}$, with $\rho_{n} \in(0,1), \forall n \in \mathbf{Z}_{0+}$. The following properties are proved:

(i) the sequence $\left\{x_{n}^{*}\right\}$ is convergent to a limit point $x^{*} \in X$ which is the unique fixed point of $T^{*}: X \rightarrow X$ which is also $\phi$-contractive;

(ii) the sequences $\left\{T_{n}^{m} x\right\}$ and $\left\{T^{* m} x\right\}$ are convergent Cauchy sequences in $X$ for any $x \in X, \forall n \in \mathbf{Z}_{0+}$, as $m$ tends to infinity.

Proof Since $\rho_{n} \leq \rho<1, \forall n \in \mathbf{Z}_{0+}, \phi: \mathbf{R} \rightarrow \mathbf{R}_{0+}$ is a $\Phi$-function and the probability density function is non-decreasing and left continuous, $F_{x, y}\left(\phi\left(\rho_{n}^{-1} t\right)\right) \geq F_{x, y}\left(\phi\left(\rho^{-1} t\right)\right), \forall t \in \mathbf{R}_{+}, \forall n \in$ $\mathbf{Z}_{0+}, \forall x, y \in X$. On the other hand,

$$
\begin{aligned}
& F_{T^{*} x, T^{*} y}(t) \\
& \geq \Delta_{M}\left(F_{T^{*} x, T_{n} x}(t / 2), F_{T^{*} y, T_{n} x}(t / 2)\right) \\
& \geq \Delta_{M}\left(F_{T^{*} x, T_{n}^{m} x}(t / 2), \Delta_{M}\left(F_{T_{n}^{m} x, T_{n}^{m_{y}}}(t / 4), F_{T^{*} y, T_{n}^{m_{y}} y}(t / 4)\right)\right) \\
& \geq \Delta_{M}\left(F_{T^{*} x, T_{n}^{m_{x}} x}(t / 4), \Delta_{M}\left(F_{T_{n}^{m_{x} x, T_{n}^{m} y}}(t / 4),\right.\right. \\
&\left.\left.F_{T^{*} y, T_{n}^{m} y}(t / 4)\right)\right), \quad \forall t \in \mathbf{R}_{+}, \forall n \in \mathbf{Z}_{0+},
\end{aligned}
$$


$\forall x, y \in X$. Define $t_{1} \in \mathbf{R}_{+}$as $t_{1}=t_{1}(t)=\left\{r \in \mathbf{R}_{+}: \phi\left(t_{1}\right)=t / 4\right\}$, which is unique since $\phi: \mathbf{R} \rightarrow$ $\mathbf{R}_{0+}$ is strictly increasing and left continuous. Since

$$
\begin{aligned}
& F_{T_{n} x, T_{n} y}\left(\phi\left(t_{1}\right)\right) \\
& \quad \geq F_{x, y}\left(\phi\left(\rho_{n}^{-1} t_{1}\right)\right) \geq F_{x, y}\left(\phi\left(\rho^{-1} t_{1}\right)\right), \quad t_{1} \in \mathbf{R}_{+}, \forall n \in \mathbf{Z}_{0+}, \forall x, y \in X, \\
& F_{T_{n}^{m} x, T_{n}^{m} y}\left(\phi\left(t_{1}\right)\right) \geq F_{x, y}\left(\phi\left(\rho^{-m} t_{1}\right)\right), \quad t_{1} \in \mathbf{R}_{+}, \forall n, m \in \mathbf{Z}_{0+}, \forall x, y \in X .
\end{aligned}
$$

Thus, there exists the $\operatorname{limit} \lim _{m \rightarrow \infty} F_{T_{n}^{m} x, T_{n}^{m} y}\left(\phi\left(t_{1}\right)\right)=F_{x, y}(\infty)=1$, then, for any given $\varepsilon \in \mathbf{R}_{+}, \lambda \in(0,1)$ and $t_{10}=t_{10}(\varepsilon)=\left\{r \in \mathbf{R}_{+}: \phi\left(t_{10}\right)=\varepsilon / 4\right\}$, there is $m_{01}=m_{01}(\varepsilon, \lambda) \in \mathbf{Z}_{0+}$ such that $F_{T_{n}^{m} x, T_{n}^{m} y}(\varepsilon / 4)>1-\lambda, \forall m\left(\in \mathbf{Z}_{0+}\right) \geq m_{01}$, so that one gets from (3.47) and (3.49):

$$
\begin{aligned}
& F_{T^{*} m_{x, T^{*}} m_{y}}(\varepsilon) \\
& \geq \Delta_{M}\left(F_{T^{*} m_{x, T_{n}^{m}}}\left(\phi\left(t_{10}\right)\right), \Delta_{M}\left(1-\lambda, F_{T^{* m}, T_{n}^{m} y} \phi\left(t_{10}\right)\right)\right), \quad \forall x, y \in X .
\end{aligned}
$$

Since $\left\{T_{n}\right\} \rightarrow T^{*}, \max \left(F_{T^{*} m_{x}, T_{n}^{m} x}\left(\phi\left(t_{10}\right)\right), F_{T^{* m}, T_{n}^{m} y}\left(\phi\left(t_{10}\right)\right)\right) \rightarrow 1$ as $m \rightarrow \infty, \forall n \in \mathbf{Z}_{0+}$, $\forall x, y \in X$, and there exists $m_{02}=m_{02}\left(\phi\left(t_{10}\right), \lambda\right) \in \mathbf{Z}_{0+}$ such that $\max \left(F_{T^{* *} m_{x}, T_{n}^{m} x}\left(\phi\left(t_{10}\right)\right)\right.$, $\left.F_{T^{* m}, T_{n}^{m} y}\left(\phi\left(t_{10}\right)\right)\right)>1-\lambda, \forall n \in \mathbf{Z}_{0+}, \forall m\left(\in \mathbf{Z}_{0+}\right) \geq m_{02}$, we have $F_{T^{*} m_{x, T^{* * m}}}\left(\phi\left(t_{10}\right)\right)>$ $1-\lambda, \forall m\left(\in \mathbf{Z}_{0+}\right) \geq m_{0}=\max \left(m_{01}, m_{02}\right)$, and the following limit exists: $\lim _{n \rightarrow \infty} F_{T^{* n} x, T^{* n} y}\left(\phi\left(t_{10}\right)\right)=1, \forall x, y \in X$, so that $\left\{T^{*^{n}} x-T^{*^{n}} y\right\} \rightarrow 0$. By choosing $y=T^{*} x$, this leads to $\lim _{n \rightarrow \infty} F_{T^{* n+1} x, T^{* n_{x}}}\left(\phi\left(t_{10}\right)\right)=1$ and $\left\{T^{*^{n}} x-T^{*^{n+1}} x\right\} \rightarrow 0, \forall x \in X$, since $\varepsilon \rightarrow 0^{+}$ implies that $\phi\left(t_{10}\right) \rightarrow 0^{+}$, since $\phi: \mathbf{R} \rightarrow \mathbf{R}_{0+}$ is a $\Phi$-function. Then $\left\{T^{*^{n}} x\right\}$ is a Cauchy sequence. Since $\left(X, F, \Delta_{M}\right)$ is complete we have $\left\{T^{*^{n}} x\right\} \rightarrow x^{*}(\in X)$ in the sense that $F_{T^{* n} n_{x, x^{*}}}(\varepsilon)>1-\lambda, \forall n\left(\in \mathbf{Z}_{0_{+}}\right) \geq n_{0}=n_{0}(\varepsilon, \lambda)$. It is now proved that such a point $x^{*}$ is a fixed point of $T^{*}: X \rightarrow X$. Assume that this is not the case so that $\left\{T^{*^{n}} x\right\} \rightarrow x^{*} \neq T x^{*}$. Then, by using a similar reasoning to previous ones, since $F_{T^{* n} x, z}(\varepsilon / 2) \rightarrow 1$ as $n \rightarrow \infty$ we have $F_{T^{* n}{ }_{n}, z}(\varepsilon / 2)>1-\lambda, \forall n \geq \bar{n}_{0}\left(\in \mathbf{Z}_{0+}\right)=\bar{n}_{0}(\varepsilon, \lambda)$ for any given $\varepsilon \in \mathbf{R}_{+}$and $\lambda \in(0,1)$. Thus there is some $\lambda_{0}=\lambda_{0}(\varepsilon) \in(0,1)$ and a strictly increasing subsequence $\left\{n_{k}\right\} \subset \mathbf{Z}_{0+}$ such that

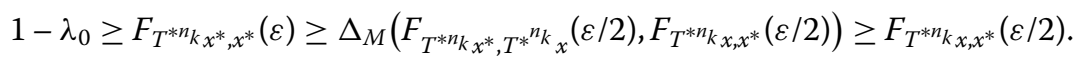

But the limit of the right-hand side convergent subsequence equals unity as that of the whole sequence, that is,

$$
\lim _{k \rightarrow \infty} F_{T^{* n_{k} x, x^{*}}}(\varepsilon / 2)=\lim _{n \rightarrow \infty} F_{T^{* n} n_{x, x^{*}}}(\varepsilon / 2)=1,
$$

which leads to the contradiction $\lambda_{0} \leq 0$ and there is no subsequence $\left\{n_{k}\right\} \subset \mathbf{Z}_{0+}$ such that $\left\{T^{*^{n}} x\right\} \rightarrow x^{*} \neq T^{n_{k}} x^{*}$ so that $x^{*} \in \operatorname{Fix}\left(T^{*}\right)$. Since it has already been proved that $\left\{T^{*^{n}} x-\right.$ $\left.T^{*^{n}} y\right\} \rightarrow 0$, the fixed point is unique so that $x^{*} \in \operatorname{Fix}\left(T^{*}\right)=\left\{x^{*}\right\}$. From the existence of the limit $\lim _{m \rightarrow \infty} F_{T_{n}^{m} x, T_{n}^{m} y}\left(\phi\left(t_{1}\right)\right)=F_{x, y}(\infty)=1$ previously proved as a consequence of (3.49), it follows that $\left\{T_{n}^{m} x-T_{n}^{m} y\right\} \rightarrow 0$ as $m \rightarrow \infty, \forall n \in \mathbf{Z}_{0+}, \forall x, y \in X$; it follows in the same way that if $\left\{T_{n}^{m} x\right\}$ is a Cauchy sequence $\forall x \in X$, then it is convergent to a limit point $x_{n}^{*}$, since $\left(X, F, \Delta_{M}\right)$ is complete and the limit point for any initial condition in $X$ is the unique fixed point of $T_{n}: X \rightarrow X, \forall n \in \mathbf{Z}_{0+}$.

It is now proved that $T^{*}: X \rightarrow X$ is $\phi$-contractive. Assume that $T^{*}: X \rightarrow X$ is not $\phi$ contractive. Then there is a sequence $\left\{x_{k}\right\} \subset X$ generated as $x_{k+1}=T^{* n_{k}} x_{k}$ with $\left\{n_{k}\right\} \subset \mathbf{Z}_{0+}$ 
and $T^{* n_{0}} x_{0} \neq x_{0} \neq x^{*}$ such that one gets for some real constant $\rho \in(0,1)$

$$
\begin{aligned}
& F_{T^{* * n_{k}} x_{k}, T^{* n_{k+1} x_{k}}}(\phi(t)) \\
& \quad=F_{T^{*} \sum_{i=0}^{k} n_{i_{x_{0}}, T^{*}} \sum_{i=0}^{k+1} n_{i_{0}}}(\phi(t)) \\
& \quad \leq F_{T^{*} \sum_{i=0}^{k-1} n_{i} x_{x_{0}}, T^{*} \sum_{i=0}^{k} n_{i} x_{x_{0}}}(\phi(t)) \leq F_{T^{* n_{0}} x_{0}, x_{0}}(\phi(t))=1-\rho<1, \quad \forall t \in \mathbf{R}_{+} .
\end{aligned}
$$

Since there exists the limit $\lim _{k \rightarrow \infty} F_{T^{*} \sum_{i=0}^{k-1} n_{i_{0}, T^{*}} \sum_{i=0}^{k} n_{i} x_{0}}(t)=F_{x^{*}, x^{*}}\left(0^{+}\right)=1, \forall t \in \mathbf{R}_{+}$, we can take limits as $k \rightarrow \infty$ in (3.53) to get the contradiction $1=F_{x^{*}, x^{*}}\left(0^{+}\right) \leq 1-\rho<1$. As a result, $T^{*}: X \rightarrow X$ is $\phi$-contractive. On the other hand, since $\operatorname{Fix}\left(T^{*}\right)=\left\{x^{*}\right\}$, there exists the limit $\lim _{n \rightarrow \infty} F_{T^{* n} x_{n}^{*}, T^{* n} x^{*}}(\phi(t))=1=F_{x^{*}, x^{*}}\left(0^{+}\right), \forall t \in \mathbf{R}_{+}$so that $\left\{x_{n}^{*}-x^{*}\right\} \rightarrow 0$.

\section{Simulation examples}

This section contains some numerical examples regarding the theoretical results introduced in the previous Section 3. The first example of [30] will be used to illustrate how the results in the deterministic case move accordingly to the probabilistic set-up. Thus, consider the iterative scheme defined by (3.1) with $T(x)=\frac{x}{2(1+x)}$ on $[0,+\infty)$ and

$$
x_{n+1}=T_{n} x_{n}=\left(\alpha_{3}^{(n)} T^{3}+\alpha_{2}^{(n)} T^{2}+\alpha_{1}^{(n)} T^{1}+\alpha_{0}^{(n)} I\right) x_{n},
$$

$\forall n \in \mathbf{Z}_{0+}$. The above description can be useful, in particular, for modeling a discretetime probabilistic system reflecting the given structure and, in that case, the $n$th running subscript index denotes successive samples. The probabilistic metric is given by $F_{x, y}(t)=\frac{t}{t+d(x, y)}$ where $d(x, y)$ is a metric, selected in this example as $d(x, y)=\|x-y\|$ where $\|\cdot\|$ stands for the Euclidean norm. Clearly, $\left([0,+\infty), F_{x, y}(t), \Delta_{M}\right)$ is a Menger PM space. Consider, firstly, the sequence of constant weights $\alpha^{(n)}=\left[\begin{array}{llll}0.2 & 0.3 & 0.8 & 0.9\end{array}\right]$ for all $n \geq 0$. We are now in a position to apply Theorem 3.1 since $\sum_{i=0}^{3} \alpha_{i}^{(n)}=2.2>0, \tilde{\alpha}_{i}^{(n)}=m_{n}=0$ for all $n \geq 0$ and (3.2) holds trivially. Furthermore, since $T$ is a strict contraction satisfying the condition $d(T(x), T(y)) \leq \rho d(x, y)$ with $\rho=1 / 2$, we have

$$
F_{x_{n+2}, x_{n+1}}(\phi(t))=\frac{\phi(t)}{\phi(t)+d\left(x_{n+2}, x_{n+1}\right)} \geq \frac{\phi(t)}{\phi(t)+\rho d\left(x_{n+1}, x_{n}\right)}=\frac{\rho^{-1} \phi(t)}{\rho^{-1} \phi(t)+d\left(x_{n+1}, x_{n}\right)},
$$

so that for $\phi(t)=t$,

$$
F_{x_{n+2}, x_{n+1}}(\phi(t)) \geq \frac{\rho^{-1} \phi(t)}{\rho^{-1} \phi(t)+d\left(x_{n+1}, x_{n}\right)}=\frac{\phi\left(\rho^{-1} t\right)}{\phi\left(\rho^{-1} t\right)+d\left(x_{n+1}, x_{n}\right)}=F_{x_{n+1}, x_{n}}\left(\phi\left(\rho^{-1} t\right)\right),
$$

and (3.3) also holds. Accordingly, the sequence of iterates $\left\{x_{n}\right\}$ is bounded for all $n \geq 0$ and converges to the unique fixed point of $T, x=0$, regardless of the initial condition $x_{0}$. These claims are verified through a numerical simulation in Figure 1.

Figure 1 shows how the iterates converge to zero, the unique fixed point of $T$, for different initial conditions. Furthermore, Figure 2 shows the behavior of the probability metric as $n$ increases. It can be noticed that, as Theorem 3.1 claims, $F_{x_{n+1}, x_{n}}(t)$ tends to unity asymptotically. 


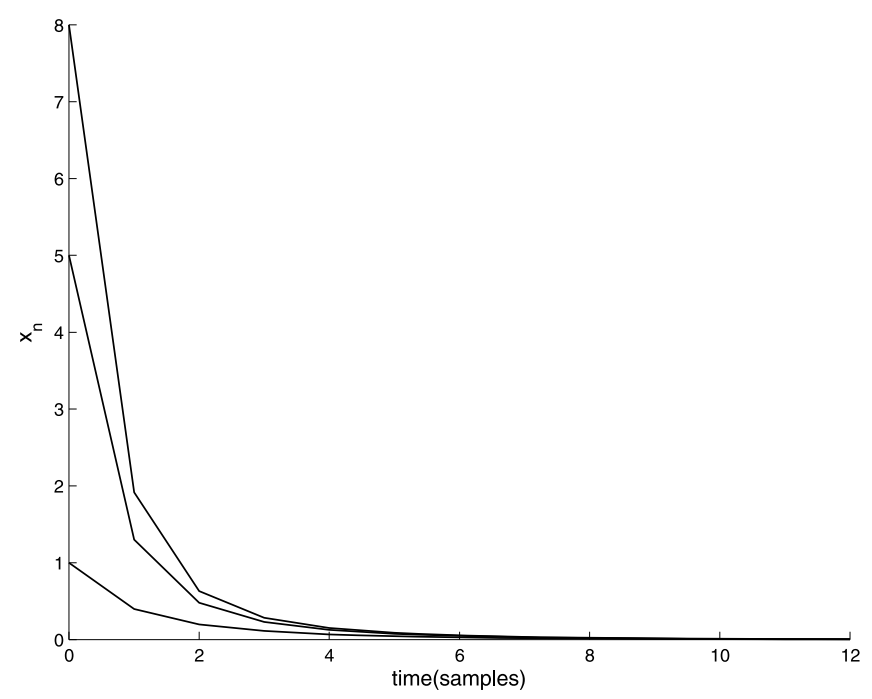

Figure 1 Evolution of the sequence of iterates for different initial conditions.

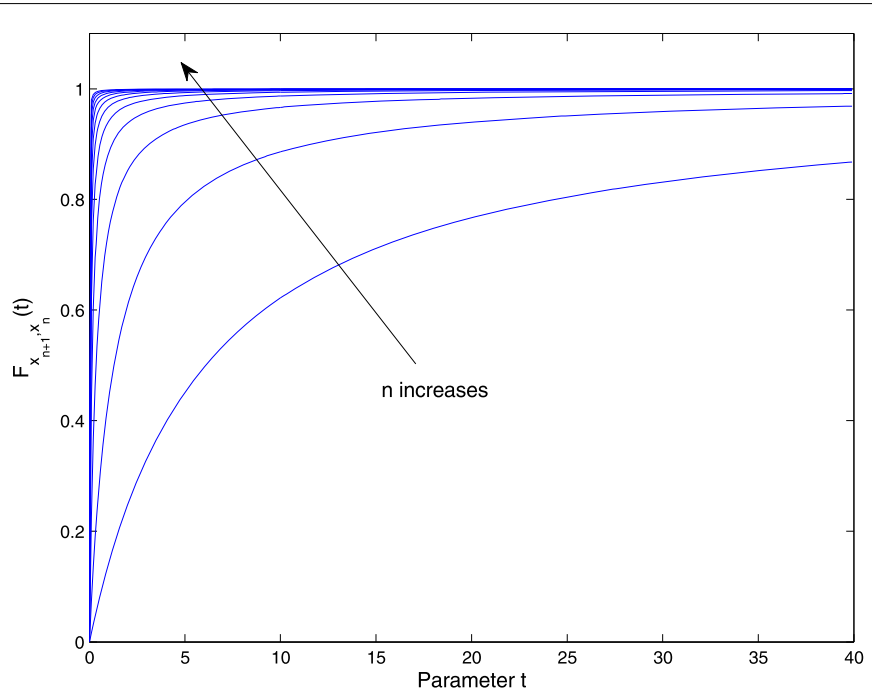

Figure 2 Evolution of the probability metric $F_{x_{n+1}, x_{n}}(t)$ as $n$ increases.

Now, we may consider the time-varying weights given by

$$
\alpha_{i}^{(n+1)}= \begin{cases}\lambda_{i} \alpha_{i}^{(n)}, & \alpha_{i}^{(n+1)} \geq 0.1 \\ 0.1, & \text { otherwise }\end{cases}
$$

for all $n \geq 0$ and $0 \leq i \leq 3$ with $\alpha^{(0)}=\left[\begin{array}{llll}0.2 & 0.3 & 0.8 & 0.9\end{array}\right], \lambda_{0}=0.95, \lambda_{1}=0.9, \lambda_{2}=0.85$ and $\lambda_{3}=0.8$. The 0.1 lower-bound has been included in (4.1) so as to satisfy the condition $\sum_{i=0}^{k} \alpha_{i}^{(n)}>0$. The evolution of the weights is depicted in Figure 3:

As it can be noted in Figure 3, the weights are decreasing with time until they reach the constant lower bound of 0.1 where they stop decreasing and become time-invariant. In 


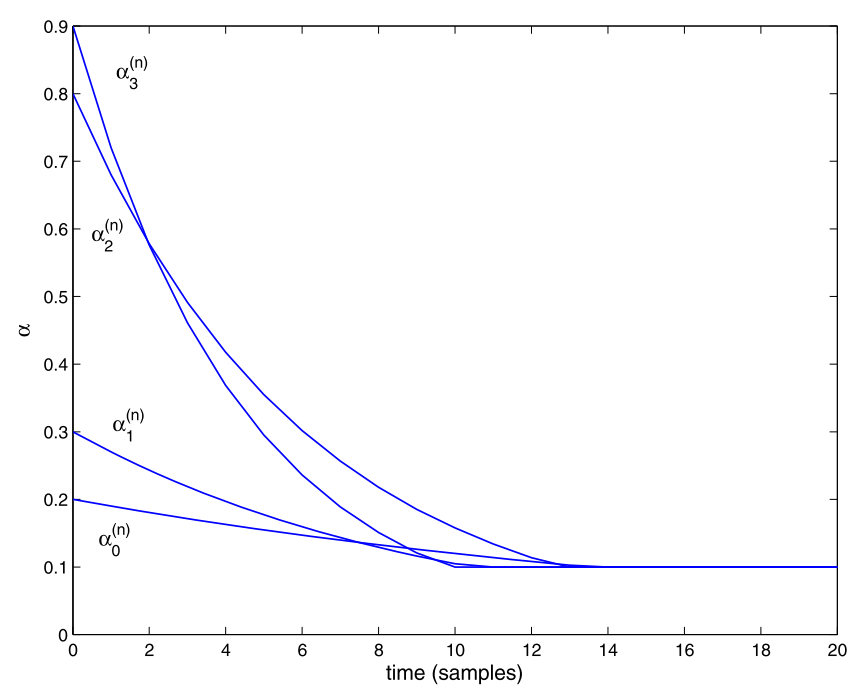

Figure 3 Evolution of the time-varying weights $\alpha_{0}^{(n)}, \alpha_{1}^{(n)}, \alpha_{2}^{(n)}$ and $\alpha_{3}^{(n)}$ through (4.1).

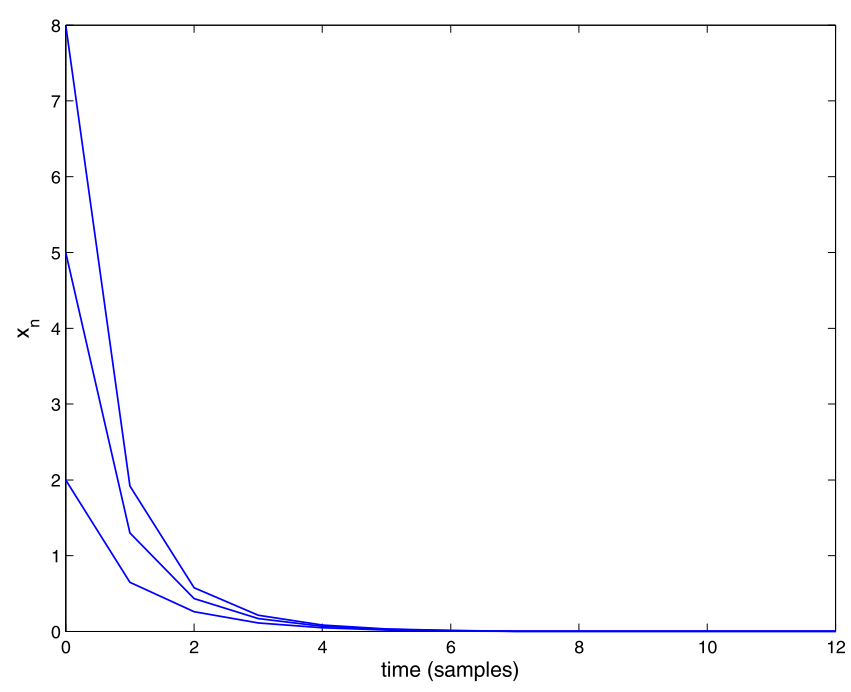

Figure 4 Iterates for different initial conditions and weights defined by (4.1).

fact, Figure 3 shows that this happens for $n \geq 14$, approximately. Moreover, notice that

$$
\begin{aligned}
\left|\tilde{\alpha}_{3}^{(1)}\right| & =\left|\alpha_{3}^{(2)}-\alpha_{3}^{(1)}\right|=\left|\lambda_{3} \alpha_{3}^{(1)}-\alpha_{3}^{(1)}\right| \\
& =\left|\lambda_{3}-1\right|\left|\alpha_{3}^{(1)}\right|=0.2 \cdot 0.9=0.18 \geq \frac{1-\rho}{k+1}=\frac{0.5}{4}=0.125
\end{aligned}
$$

so that (3.2) of Theorem 3.1 no longer holds. However, since the weights converge to a finite limit, the operator sequence $T_{n}$ converges to a constant one $T^{*}$ and we are in a position to apply Theorem 3.3. Therefore, as Figure 4 displays, the sequence of iterates is bounded and converges to the unique fixed point of $T^{*}$, which is $x=0$.

One of the main advantages of the results established in Theorem 3.3 is that it is not necessary to satisfy the conditions of Theorem 3.1 for all samples since a potentially arbitrary 


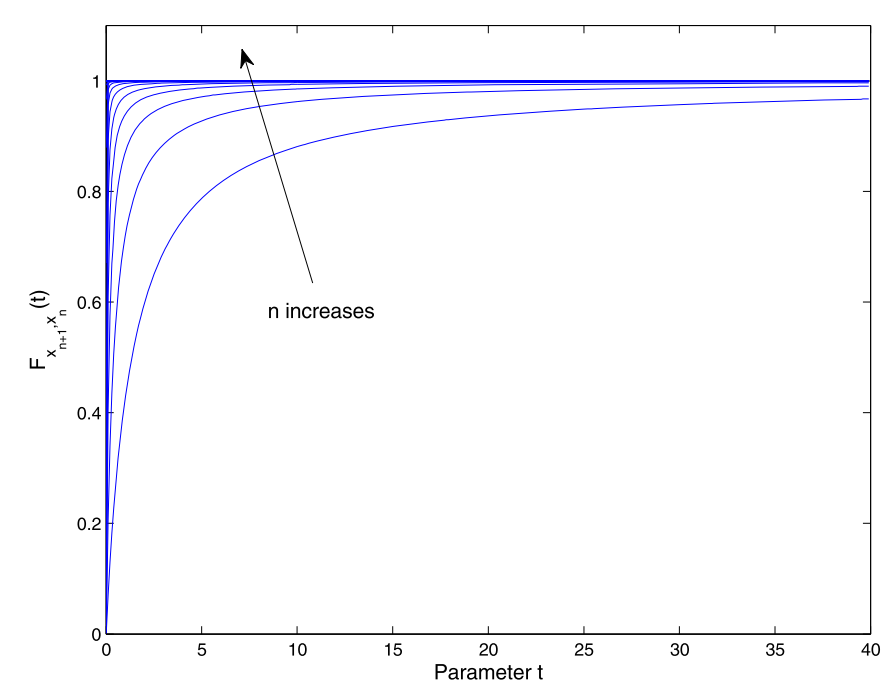

Figure 5 Evolution of the probability metric as $n$ increases.

variation in the weights is admitted on certain subsets of the natural numbers by relaxing the contractive constraint on the operator. Therefore, the family of admissible time variations for which the stability of the iteration scheme is guaranteed is enlarged with respect to other approaches. Finally, Figure 5 shows the time evolution of the probability metric. As can be seen in Figure 5, the probability metric tends asymptotically to unity again as Theorem 3.3 holds.

Competing interests

The authors declare that they have no competing interests.

Authors' contributions

Both authors have contributed equally to this manuscript.

\section{Author details}

${ }^{1}$ Instituto of Research and Development of Processes, University of the Basque Country Campus of Leioa (Bizkaia), Barrio Sarriena, P.O. Box 644, Bilbao, Leioa 48940, Spain. Department of Telecommunications and Systems Engineering, Universitat Autònoma de Barcelona, UAB, Barcelona 08193, Spain.

\section{Acknowledgements}

The authors are very grateful to the Spanish and Basque Governments and to UPV/EHU for Grants DPI2012-30651, DPI2013-47825-C3-1-R, IT378-10, SAIOTEK S-PE13UN039 and UFI 2011/07. The authors are also very grateful to the reviewers for their useful comments.

Received: 17 December 2014 Accepted: 16 July 2015 Published online: 05 August 2015

\section{References}

1. Schweizer, B, Sklar, A: Probabilistic Metric Spaces. North-Holland, Amsterdam (1983)

2. Pap, E, Hadzic, O, Mesiar, R: A fixed point theorem in probabilistic metric spaces and an application. J. Math. Anal. Appl. 202, 433-439 (1996)

3. Sehgal, VM, Bharucha-Reid, AT: Fixed points of contraction mappings on probabilistic metric spaces. Theory Comput. Syst. 6(1), 97-102 (1972)

4. Schweizer, B, Sklar, A: Statistical metric spaces. Pac. J. Math. 10, 313-334 (1960)

5. Choudhury, BS, Das, K, Bhandari, SK: Fixed point theorem for mappings with cyclic contraction in Menger spaces. Int. J. Pure Appl. Sci. Technol. 4(1), 1-9 (2011)

6. Choudhury, BS, Das, K, Bhandari, SK: Cyclic contraction result in 2-Menger space. Bull. Int. Math. Virtual Inst. 2(1), 223-234 (2012)

7. Beg, I, Abbas, M: Fixed point and best approximation in Menger convex metric spaces. Arch. Math. 41, 389-397 (2005)

8. Beg, I, Latif, A, Abbas, M: Coupled fixed points of mixed monotone operators on probabilistic Banach spaces. Arch. Math. 37, 1-8 (2001)

9. Mihet, D: Altering distances in probabilistic Menger spaces. Nonlinear Anal. 71, 2734-2738 (2009) 
10. Mihet, D: A Banach contraction theorem for fuzzy metric spaces. Fuzzy Sets Syst. 144, 431-439 (2004)

11. Sedghi, S, Choudhury, BS, Shobe, N: Strong common coupled fixed point result in fuzzy metric spaces. J. Phys. Sci. 17, $1-9$ (2013)

12. Choudhury, BS, Das, KP: A new contraction principle in Menger spaces. Acta Math. Sin. Engl. Ser. 24(8), 1379-1386 (2008)

13. Gopal, D, Abbas, M, Vetro, C: Some new fixed point theorems in Menger PM-spaces with application to Volterra type integral equation. Appl. Math. Comput. 232, 955-967 (2014)

14. Khan, MS, Swaleh, M, Sessa, S: Fixed point theorems by altering distances between the points. Bull. Aust. Math. Soc 30(1), 1-9 (1984)

15. Eldred, AA, Veeramani, P: Existence and convergence of best proximity points. J. Math. Anal. Appl. 323, $1001-1006$ (2006)

16. De la Sen, M: Linking contractive self-mappings and cyclic Meir-Keeler contractions with Kannan self-mappings. Fixed Point Theory Appl. 2010, Article ID 572057 (2010). doi:10.1155/2010/572057

17. De la Sen, M, Agarwal, RP, Nistal, R: Non-expansive and potentially expansive properties of two modified p-cyclic self-maps in metric spaces. J. Nonlinear Convex Anal. 14(4), 661-686 (2013)

18. De la Sen, M, Agarwal, RP: Fixed point-type results for a class of extended cyclic self-mappings under three general weak contractive conditions of rational type. Fixed Point Theory Appl. 2011, Article ID 102 (2011). doi:10.1186/1687-1812-2011-102

19. De la Sen, M: On best proximity point theorems and fixed point theorems for $p$-cyclic hybrid self-mappings in Banach spaces. Abstr. Appl. Anal. 2013, Article ID 183174 (2013). doi:10.1155/2013/183174

20. Karpagam, S, Agrawal, S: Best proximity point theorems for $p$-cyclic Meir-Keeler contractions. Fixed Point Theory Appl. 2009, Article ID 197308 (2009)

21. Suzuki, T: Some notes on Meir-Keeler contractions and L-functions. Bull. Kyushu Inst. Technol. 53, 12-13 (2006)

22. Di Bari, C, Suzuki, T, Vetro, C: Best proximity points for cyclic Meir-Keeler contractions. Nonlinear Anal. 69(11), 2790-3794 (2008)

23. Rezapour, S, Derafshpour, M, Shahzad, N: On the existence of best proximity points of cyclic contractions. Adv. Dyn. Syst. Appl. 6(1), 33-40 (2011)

24. Derafshpour, M, Rezapour, S, Shahzad, N: Best proximity points of cyclic $\boldsymbol{\phi}$-contractions on reflexive Banach spaces. Fixed Point Theory Appl. 2010, Article ID 946178 (2010)

25. Al-Thagafi, MA, Shahzad, S: Convergence and existence results for best proximity points. Nonlinear Anal. 70(10), 3665-3671 (2009)

26. Delasen, M: Stability of composite systems with and asymptotically hyperstable subsystem. Int. J. Control 44(6), 1769-1775 (1986)

27. Garrido, I, Garrido, AJ, Sevillano, M, Romero, JA: Robust sliding mode control for tokamaks. Math. Probl. Eng. 2012, Article ID 341405 (2012)

28. De la Sen, M, Garrido, AJ, Soto, JC, Barambones, O: Suboptimal regulation for a class of bilinear interconnected systems with finite-time sliding planning horizons. Math. Probl. Eng. 2008, Article ID 817063 (2008)

29. Delasen, M: Application of the non-periodic sampling to the identifiability and model-matching problems in dynamic systems. Int. J. Syst. Sci. 14(4), 367-383 (1983)

30. De la Sen, M, Ibeas, A: Convergence properties and fixed points of two general iterative schemes with composed maps in Banach spaces with applications to guaranteed global stability. Abstr. Appl. Anal. 2014, Article ID 948749 (2014)

\section{Submit your manuscript to a SpringerOpen ${ }^{\circ}$ journal and benefit from:}

- Convenient online submission

Rigorous peer review

- Immediate publication on acceptance

- Open access: articles freely available online

- High visibility within the field

- Retaining the copyright to your article 